ESAIM: M2AN

Vol. 40, No 3 , 2006, pp. 597-621

DOI: $10.1051 / \mathrm{m} 2 \mathrm{an}: 2006025$
ESAIM: Mathematical Modelling and Numerical Analysis www.edpsciences.org/m2an

\title{
LARGE TIME BEHAVIOR OF SOLUTIONS IN SUPER-CRITICAL CASES TO DEGENERATE KELLER-SEGEL SYSTEMS
}

\author{
Stephan Luckhaus ${ }^{1}$ And Yoshie Sugiyama ${ }^{2}$
}

Abstract. We consider the following reaction-diffusion equation:

$$
(\mathrm{KS}) \begin{cases}u_{t}=\nabla \cdot\left(\nabla u^{m}-u^{q-1} \nabla v\right), & x \in \mathbb{R}^{N}, 0<t<\infty, \\ 0=\Delta v-v+u, & x \in \mathbb{R}^{N}, 0<t<\infty, \\ u(x, 0)=u_{0}(x), & x \in \mathbb{R}^{N},\end{cases}
$$

where $N \geq 1, m>1, q \geq \max \left\{m+\frac{2}{N}, 2\right\}$.

In [Sugiyama, Nonlinear Anal. 63 (2005) 1051-1062; Submitted; J. Differential Equations (in press)] it was shown that in the case of $q \geq \max \left\{m+\frac{2}{N}, 2\right\}$, the above problem (KS) is solvable globally in time for "small $L^{\frac{N(q-m)}{2}}$ data". Moreover, the decay of the solution $(u, v)$ in $L^{p}\left(\mathbb{R}^{N}\right)$ was proved. In this paper, we consider the case of " $q \geq \max \left\{m+\frac{2}{N}, 2\right\}$ and small $L^{\ell}$ data" with any fixed $\ell \geq \frac{N(q-m)}{2}$ and show that (i) there exists a time global solution $(u, v)$ of $(\mathrm{KS})$ and it decays to 0 as $t$ tends to $\infty$ and (ii) a solution $u$ of the first equation in (KS) behaves like the Barenblatt solution asymptotically as $t$ tends to $\infty$, where the Barenblatt solution is the exact solution (with self-similarity) of the porous medium equation $u_{t}=\Delta u^{m}$ with $m>1$.

Mathematics Subject Classification. 35B40, 35K45, 35K55, 35k65.

Received: September 22, 2005. Revised: February 27, 2006.

\section{INTRODUCTION}

We consider the following reaction-diffusion equation:

$$
\begin{cases}u_{t}=\nabla \cdot\left(\nabla u^{m}-u^{q-1} \nabla v\right), & x \in \mathbb{R}^{N}, 0<t<\infty \\ 0=\Delta v-v+u, & x \in \mathbb{R}^{N}, 0<t<\infty \\ u(x, 0)=u_{0}(x), & x \in \mathbb{R}^{N}\end{cases}
$$

Keywords and phrases. Degenerate parabolic system, chemotaxis, Keller-Segel model, drift term, decay property, asymptotic behavior, Fujita exponent, porous medium equation, Barenblatt solution.

${ }^{1}$ Departement of mathematics and computer science, Universität Leipzig, Leipzig, 04109, Germany. luckhaus@mis.mpg.de

2 Department of Mathematics and Computer Science, Tsuda College, 2-1-1, Tsuda-chou, Kodaira-shi, Tokyo, 187-8577, Japan. sugiyama@tsuda.ac.jp

(c) EDP Sciences, SMAI 2006 
where $N \geq 1, m>1, q \geq \max \left\{m+\frac{2}{N}, 2\right\}$. The initial data $u_{0}$ is a non-negative function in $L^{1} \cap L^{\infty}\left(\mathbb{R}^{N}\right) \times L^{1} \cap$ $H^{1} \cap W^{1, \infty}\left(\mathbb{R}^{N}\right), u_{0}^{m} \in H^{1}\left(\mathbb{R}^{N}\right)$. This equation is often called the Keller-Segel model describing the motion of the chemotaxis molds. (We refer to Keller-Segel [23].)

In this paper, we are interested in the large time behavior of solutions for (KS). Concerning the large time behavior of the heat equation, the following asymptotic profile is well known:

$$
\lim _{t \rightarrow \infty} t^{\frac{N}{2}}\left\|u(\cdot, t)-M G_{t}(\cdot)\right\|_{L^{\infty}\left(\mathbf{R}^{N}\right)}=0
$$

where $G_{t}(x)$ is the heat kernel and $M$ is the initial mass.

Also for the porous medium equation:

$$
u_{t}(x, t)=\Delta u^{m}(x, t)
$$

corresponding to the initial data $u_{0}$, the asymptotic profile was obtained in the following form:

$$
\lim _{t \rightarrow \infty} t^{\sigma}\left\|u(\cdot, t)-V\left(\cdot, t ;\left\|u_{0}\right\|_{L^{1}\left(\mathbf{R}^{N}\right)}\right)\right\|_{L^{\infty}\left(\mathbf{R}^{N}\right)}=0 \quad \text { with } \sigma=\frac{N}{N(m-1)+2},
$$

where $V(x, t ; M)$ is the exact solution of $(\mathrm{P})$ given by

$$
V(x, t ; M):=\frac{1}{t^{\sigma}}\left(\beta^{2} M^{\frac{2 \sigma(m-1)}{N}}-\frac{\sigma(m-1)}{2 m N} \cdot \frac{|x|^{2}}{t^{\frac{2 \sigma}{N}}}\right)_{+}^{\frac{1}{m-1}}
$$

with a constant $\beta$ such that $\int_{\mathbb{R}^{N}}\left(\beta^{2}-\frac{\sigma(m-1)}{2 m N}|y|^{2}\right)_{+}^{\frac{1}{m-1}} \mathrm{~d} y=1$. The above $V(x, t ; M)$ holds the self-similarity and $\int_{\mathbb{R}^{N}} V(x, t ; M) \mathrm{d} x=M$ for all $t>0$. This $V(x, t ; M)$ is called the Barenblatt solution. (See Barenblatt [2].) The asymptotic profile (1.2) was firstly proved by Kamin [20], and developed by Friedman-Kamin [10], and finally established by Vazquez [34] in the above form (1.3). (We also refer to [3,21,35].)

Regarding to the Keller-Segel model (KS), for the semilinear case: $m=1$ of parabolic-parabolic type, Nagai-Syukuinn-Umesako [26] showed the similar asymptotic profile to (1.1). (we also refer to Biler-CannoneGuerra-Karch [5].) Their argument is based on the representation formula. On the other hand, as for our problem (KS), there is no representation formula for solution $u$ since $m>1$. In addition, differently from the porous medium equation (P), comparison principles do not hold. Therefore, we can not employ the method by Kamin [20], Friedman-Kamin [10], Nagai-Syukuinn-Umesako [26] to our problem directly.

Our aim of this paper is to prove the following (I)-(III) without "comparison principles and representation formula":

In the case of $m>1$ and $q \geq \max \left\{m+\frac{2}{N}, 2\right\}$;

(I) (KS) is globally solvable for the small $L^{\ell}$ data with any fixed $\ell \geq \frac{N(q-m)}{2}$;

(II) the solution $(u, v)$ of $(\mathrm{KS})$ decays to 0 in $L^{p}\left(\mathbb{R}^{N}\right)(1<p<\infty)$.

We also assume that $q>m+\frac{2}{N}$. Then,

(III) the solution $u$ to the first equation in (KS) satisfies the following asymptotic profile:

$$
\lim _{t \rightarrow \infty} t^{\frac{N}{N(m-1)+2+\varepsilon}\left(1-\frac{1}{p}\right)} \| u(\cdot, t)-V\left(\cdot, t ;\left\|u_{0}\right\|_{\left.L^{1}\right)} \|_{L^{p}\left(B_{t}\right)}=0, \quad \varepsilon>0 \text { and } 1<p<\infty\right.
$$

for all $R>0$, where $B_{t}=B_{t}(\varepsilon, R):=\left\{x \in \mathbb{R}^{N} ;|x| \leq R t^{\frac{1}{N(m-1)+2+\varepsilon}}\right\}$. 
In what follows, we give the definition of a weak solution $(u, v)$ for $(\mathrm{KS})$.

Definition 1. Let $m>1 q \geq 2$ and let $u_{0} \in L^{1} \cap L^{\infty}\left(\mathbb{R}^{N}\right)$ with $u_{0}^{m} \in H^{1}\left(\mathbb{R}^{N}\right)$ and $u_{0} \geq 0$. A pair $(u, v)$ of non-negative functions defined in $\mathbb{R}^{N} \times[0, T)$ is called a weak solution of (KS) on $[0, T)$ if

(i) $u \in L^{\infty}\left(0, T ; L^{1} \cap L^{\infty}\left(\mathbb{R}^{N}\right)\right), \nabla u^{m} \in L^{2}\left(0, T ; L^{2}\left(\mathbb{R}^{N}\right)\right)$;

(ii) $v \in L^{\infty}\left(0, T ; H^{1}\left(\mathbb{R}^{N}\right)\right)$;

(iii) $(u, v)$ satisfies the equations in the sense of distribution, i.e., that

$$
\begin{aligned}
& \int_{0}^{\infty} \int_{\mathbb{R}^{N}}\left(\nabla u^{m} \cdot \nabla \varphi-u^{q-1} \nabla v \cdot \nabla \varphi-u \varphi_{t}\right) \mathrm{d} x \mathrm{~d} t=\int_{\mathbf{R}^{N}} u_{0}(x) \varphi(x, 0) \mathrm{d} x \\
& \int_{\mathbb{R}^{N}}(\nabla v \cdot \nabla \psi+v \psi-u \psi)(t) \mathrm{d} x=0 \quad \text { for a.a. } t \in(0, T)
\end{aligned}
$$

for all functions $\varphi \in C_{0}^{\infty}\left(\mathbb{R}^{N} \times[0, T)\right)$ and $\psi \in C_{0}^{\infty}\left(\mathbb{R}^{N}\right)$.

In the first theorem, we show the existence and decay property of a solution $(u, v)$ for $(\mathrm{KS})$ with small initial data.

Theorem 1.1 (decay property). Let $1 \leq p<\infty, N \geq 1, m>1, q \geq \max \left\{m+\frac{2}{N}, 2\right\}, \ell \geq \frac{N(q-m)}{2}(\geq 1)$. Suppose that the initial data $u_{0}$ is non-negative everywhere. Then, there exist an absolute constant $M$ and $a$ positive number $\varepsilon$ depending only on $M, p, N, m, \ell$ such that if $u_{0} \in L^{1} \cap L^{\ell}\left(\mathbb{R}^{N}\right)$ satisfies that

$$
\left\|u_{0}\right\|_{L^{1}\left(\mathbf{R}^{N}\right)}=M, \quad\left\|u_{0}\right\|_{L^{\ell}\left(\mathbf{R}^{N}\right)} \leq \varepsilon
$$

then (KS) has a weak solution $(u, v)$ on $[0, \infty)$ with the following decay property: there exists a constant $C_{p}$ depending only on $p,\left\|u_{0}\right\|_{L^{p}\left(\mathbf{R}^{N}\right)}$ together with $N, m, q, M,\left\|u_{0}\right\|_{L^{(N+2) q}\left(\mathbf{R}^{N}\right)}$ such that

$$
\|u(t)\|_{L^{p}\left(\mathbf{R}^{N}\right)}+\|v(t)\|_{L^{p}\left(\mathbf{R}^{N}\right)} \leq C_{p}(1+t)^{-d} \text { for all } 0<t<\infty,
$$

where

$$
d=\sigma\left(1-\frac{1}{p}\right), \quad \sigma=\frac{N}{N(m-1)+2}
$$

\section{Remark 1.}

(i) The decay rate $d$ depends on $m, N$ but not on $q$.

(ii) The above decay rate seems to be optimal. In fact, for $m=1$, we find that $\sigma=\frac{N}{2}$ whose decay rate $d$ coincides with the $L^{1}-L^{p}$ estimate for the linear heat equation.

(iii) Concerning the following Cauchy problem

$$
\text { (PS) } \begin{cases}u_{t}=\Delta u^{m}+u^{q} & x \in \mathbb{R}^{N}, t>0 \\ u(x, 0)=u_{0}(x), & x \in \mathbb{R}^{N}\end{cases}
$$

in the case of " $m \geq 1$ and $q>m+\frac{2}{N}$ ", Kawanago [22] obtained the decay estimate under smallness assumption for $\left\|u_{0}\right\|_{L} \frac{N(q-m)}{2}$. In Remark 4.1 in [22], he mentioned that $p_{0}:=\frac{N(q-m)}{2}$ is the special exponent to obtain the decay property for (PS). Regarding to (KS), we show that if $\left\|u_{0}\right\|_{L^{\ell}\left(\mathbf{R}^{N}\right)}<<1$ for any fixed number $\ell \geq \frac{N(q-m)}{2}(\geq 1)$, then the decay property is obtained, i.e., that the exponent $p_{0}$ is not special for $(\mathrm{KS})$.

For any positive numbers $\varepsilon, R$, we define $B_{t}$ by

$$
B_{t}:=\left\{x \in \mathbb{R}^{N} ;|x|<R t^{\frac{1}{N(m-1)+2+\varepsilon}}\right\} .
$$


We introduce the self-similar solution $V(x, t ; M)$ of Barenblatt [2]:

$$
\begin{aligned}
& V(x, t ; M):=\frac{1}{t^{\sigma}}\left(\beta^{2} M^{\frac{2 \sigma(m-1)}{N}}-\frac{\sigma(m-1)}{2 m N} \cdot \frac{|x|^{2}}{\left.t^{\frac{2 \sigma}{N}}\right)_{+}^{\frac{1}{m-1}}}\right. \\
& \quad \text { with a constant } \beta \text { such that } \int_{\mathbb{R}^{N}}\left(\beta^{2}-\frac{\sigma(m-1)}{2 m N}|y|^{2}\right)_{+}^{\frac{1}{m-1}} \mathrm{~d} y=1 .
\end{aligned}
$$

It is easily verified that

$$
\int_{\mathbf{R}^{N}} V(x, t ; M) \mathrm{d} x=M .
$$

We now give the asymptotic profile in the following theorem.

Theorem 1.2 (asymptotic profile). Let the same assumption as that in Theorem 1.1 hold. In addition, let $q>m+\frac{2}{N}$. Then, the weak solution u obtained in Theorem 1.1 satisfies that

$$
\lim _{t \rightarrow \infty} t^{\frac{N}{N(m-1)+2+\varepsilon}\left(1-\frac{1}{p}\right)}\left\|u(\cdot, t)-V\left(\cdot, t ;\left\|u_{0}\right\|_{L^{1}\left(\mathbf{R}^{N}\right)}\right)\right\|_{L^{p}\left(B_{t}\right)}=0 \quad \text { with } \varepsilon>0,1<p<\infty,
$$

for all $R>0$, where $B_{t}=B_{t}(\varepsilon, R)$ is the ball defined in (1.7).

\section{Remark 2.}

(i) It seems to be difficult to take $\varepsilon=0$.

(ii) Theorem 1.2 implies that $\Delta u^{m}$ is dominant to $\nabla\left(u^{q-1} \nabla v\right)$ in the case of " $q>m+\frac{2}{N}$ and small initial data".

(iii) The proof of Theorem 1.2 is based on the estimate (1.6) in Theorem 1.1.

To show the asymptotic profile for (KS) with $m>1$, we consider the following sequence of functions:

$$
w_{k}(x, t)=k^{N} u\left(k x, k^{N(m-1)+2} t\right) \quad \text { and } \quad z_{k}(x, t)=k^{N} v\left(k x, k^{N(m-1)+2} t\right) \quad \text { for } k \geq 1 .
$$

Then, (KS) can be rewritten as follows:

$$
{ }^{w}(\mathrm{KS})\left\{\begin{array}{rlrl}
w_{k t}(x, t) & =\nabla \cdot\left(\nabla\left(w_{k}\right)^{m}-k^{-N(q-m)} \cdot\left(w_{k}\right)^{q-1} \cdot \nabla z_{k}\right), & x \in \mathbb{R}^{N}, 0<t<\infty, & \cdots(1)_{w}, \\
0 & =\Delta z_{k}-k^{2} z_{k}+k^{2} w_{k}, & x \in \mathbb{R}^{N}, 0<t<\infty, \quad \cdots(2)_{w}, \\
w_{k}(x, 0) & =k^{N} u_{0}(k x), & x \in \mathbb{R}^{N} .
\end{array}\right.
$$

The above system ${ }^{w}(\mathrm{KS})$ does not have any invariance under change of scaling. In addition, the second equation includes the scaling parameter $k$. However, in the case of $q \geq m+\frac{2}{N}$, we obtain the $L^{\infty}\left(\mathbb{R}^{N}\right)$-bound for $w_{k}$ independently of $k$. Next, we prove that $\left(w_{k}\right)^{m}$ is bounded in $H^{1}\left(\delta, T ; L^{2}\left(\mathbb{R}^{N}\right)\right) \cap L^{\infty}\left(\delta, T ; H^{1}\left(\mathbb{R}^{N}\right)\right)$ for all $0<\delta<T<\infty$. In this point, we have the difficulty such as $\left\|w_{k}(0)\right\|_{L^{p}\left(\mathbb{R}^{N}\right)}=\left(k^{N\left(1-\frac{1}{p}\right)}\left\|u_{0}\right\|_{L^{p}\left(\mathbf{R}^{N}\right)}\right)$ depends on $k$ for all $p \in(1, \infty]$. Under this difficulty, to obtain the boundness in $H^{1}\left(\delta, T ; L^{2}\left(\mathbb{R}^{N}\right)\right) \cap L^{\infty}\left(\delta, T ; H^{1}\left(\mathbb{R}^{N}\right)\right)$ independent of $k$, we use the cut-off function which attains 0 at $t=0$ and has $C^{\infty}$-regularity. As a result, we obtain the desired bounds independent of $k$ (see Sect. 5.1 in this paper) and show that $w_{k}$ converges a function $U$. Simultaneously, we find that $U$ satisfies (P) in a distribution sense since the power of $k$ in the coefficient of the perturbation term is negative. (See Sect. 5.2)

Furthermore, we verify the following key fact:

$$
U(\cdot, t) \in L^{1}\left(\mathbb{R}^{N}\right) \quad \text { and } \quad\|U(\cdot, t)\|_{L^{1}\left(\mathbf{R}^{N}\right)}=\left\|u_{0}\right\|_{L^{1}\left(\mathbf{R}^{N}\right)} .
$$


To this end, we invent the crucial lemma (Lem. 2.4) in Section 2:

$$
\text { " } w_{k} \rightarrow U \quad \text { strongly in } L_{l o c}^{1}\left(\mathbb{R}^{N}\right)
$$

together with some additional assumption on $w_{k}$, we have in fact, that

$$
\left|\int_{\mathbf{R}^{N}}\left(w_{k}-U\right) \mathrm{d} x\right| \rightarrow 0 \quad \text { as } k \rightarrow \infty . "
$$

We ensure the sufficient conditions in the above lemma (Lem. 2.4). (See Sect. 5.3.) As a result, by virtue of (1.11) and "the mass conservation law and the $L^{1}$-scaling invariance for the initial data of $w_{k}$ ", i.e., that

$$
\int_{\mathbf{R}^{N}} w_{k}(x, t) \mathrm{d} x=\int_{\mathbb{R}^{N}} w_{k}(x, 0) \mathrm{d} x=\int_{\mathbb{R}^{N}} u_{0} \mathrm{~d} x \quad \text { for all } t \geq 0,
$$

the above $(\mathrm{H})$ is verified. Once $(\mathrm{H})$ is verified, we can apply Theorem 1.1 in Vazquez [34] and obtain that

$$
\left\|U(\cdot, t)-V\left(\cdot, t ;\left\|u_{0}\right\|_{L^{1}}\right)\right\|_{L^{p}\left(\mathbb{R}^{N}\right)} \rightarrow 0 \quad \text { as } t \rightarrow \infty
$$

For any positive numbers $\varepsilon, R$, we define $B_{t}$ by

$$
B_{t}:=\left\{x \in \mathbb{R}^{N} ;|x|<R t^{\frac{1}{N(m-1)+2+\varepsilon}}\right\}
$$

Then, taking the time variable by $k^{\varepsilon}$, and combining (1.13) with the convergence of $w_{k}$ to $U$, we observe that

$$
\begin{aligned}
& \left\|w_{k}\left(\cdot, k^{\varepsilon}\right)-V\left(\cdot, k^{\varepsilon} ;\left\|u_{0}\right\|_{L^{1}}\right)\right\|_{L^{p}\left(B_{R}\right)} \\
& \leq\left\|w_{k}\left(\cdot, k^{\varepsilon}\right)-U\left(\cdot, k^{\varepsilon}\right)\right\|_{L^{p}\left(B_{R}\right)}+\left\|U\left(\cdot, k^{\varepsilon}\right)-V\left(\cdot, k^{\varepsilon} ;\left\|u_{0}\right\|_{L^{1}}\right)\right\|_{L^{p}\left(\mathbb{R}^{N}\right)} \\
& \rightarrow 0 \quad \text { as } k \rightarrow \infty
\end{aligned}
$$

for any $p \in(1, \infty)$ and for all $R>0$, where $B_{R}:=\left\{x \in \mathbb{R}^{N} ;|x|<R\right\}$. Moreover, taking $k$ by $k=t^{\frac{1}{N(m-1)+2+\varepsilon}}$ in (1.15) and using the self-similarity of the Barenblatt solution, we conclude that

$$
t^{\frac{N}{N(m-1)+2+\varepsilon}\left(1-\frac{1}{p}\right)}\left\|u(\cdot, t)-V\left(\cdot, t ;\left\|u_{0}\right\|_{L^{1}}\right)\right\|_{L^{p}\left(B_{t}\right)} \rightarrow 0 \text { as } t \rightarrow \infty
$$

for any $\varepsilon>0$ and $p \in(1, \infty)$ and for all $R>0$, where $B_{t}=B_{t}(\varepsilon, R)$ is the ball defined in (1.14). Thus, we prove Theorem 1.2. (see Section 5.4.)

In the following section, we shall prepare several lemmas which will be used in the sequent sections. In Section 3, we introduce the results obtained in [30-32] concerning the existence of a time global strong solution of the approximated problem of (KS). In Section 4, we organize the proof of the decay of a solution $(u, v)$. In Section 5 , in the case of " $m>1, q>m+\frac{2}{N}$ ", we prove that the solution $u$ of $(\mathrm{KS})$ behaves like the Barenblatt solution asymptotically as $t \rightarrow \infty$ which is the exact solution of porous medium equation: $u_{t}=\Delta u^{m}$ with $m>1$. 


\section{Remark 3.}

(i) In our argument, any type of comparison principles is not used.

(ii) When we substitute the second equation: $\Delta v=v-u$ into the first equation in (KS), it holds that

$$
\text { (E) } u_{t}=\Delta u^{m}-\nabla u^{q-1} \cdot \nabla v-u^{q-1} \Delta v=\Delta u^{m}+u^{q}-\nabla u \cdot \nabla v-u^{q-1} v \text {. }
$$

The above equation (E) includes the terms $u_{t}, \Delta u^{m}$ and $u^{q}$. Therefore, we observe that (PS) in Remark 1 is analogous to $(\mathrm{E})$.

For (PS) with $N \geq 1, m, q>1$, it is well known that the critical exponent $q=m+\frac{2}{N}$ divides the situation into the global existence and the finite time blow-up of a solution. Indeed,

(1) when $q>m+\frac{2}{N}$, the problem (PS) is globally solvable for small initial data and evolves in a finite time blow-up for large initial data and

(2) when $q<m+\frac{2}{N}$ and $q=m+\frac{2}{N}$, it is proved that (all) non-negative solutions of (PS) blow up in a finite time without any restriction on the size of the initial data. (See for example Galaktionov-KurdyumovMikhailov-SamarskiiN [13], Galaktionov [12], Kawanago [22] and Mochizuki-Suzuki[24].) This exponent $q=m+\frac{2}{N}$ is called the Fujita exponent [11].

For (KS) with $N \geq 1, m>1, q \geq 2$, in [30-32] the Fujita's exponent was found. Specifically, in [32] it was shown that

(i) when $q<m+\frac{2}{N}$, the problem (KS) is globally solvable without any restriction on the size of the initial data; and

(ii) when $m>1$ and $q \geq \max \left\{m+\frac{2}{N}, 2\right\}$, the problem $(\mathrm{KS})$ is globally solvable for small $L^{\frac{N(q-m)}{2}}$ initial data. Furthermore, the decay of solution $(u, v)$ in $L^{p}\left(\mathbb{R}^{N}\right)(1<p<\infty)$ was proved.

In addition, in the case of $q=2$ with $2>m+\frac{2}{N}$;

(iii) we [33] constructed such an initial function that a solution $(u, v)$ blows up in a finite time.

In this paper, the case of (ii) above is considered.

We will use the simplified notations:

(1) $\partial_{t}=\frac{\partial}{\partial t}, \quad \partial_{i}=\frac{\partial}{\partial x_{i}}, \partial_{i j}^{2}=\partial_{i} \partial_{j}, \nabla u=\left(\partial_{1}, \partial_{2}, \cdots\right), \nabla^{2} u=\left(\partial_{11}^{2}, \partial_{12}^{2}, \cdots\right)$;

(2) $\|\cdot\|_{L^{r}}=\|\cdot\|_{L^{r}\left(\mathbb{R}^{N}\right)},(1 \leq r \leq \infty), \int \cdot \mathrm{d} x:=\int_{\mathbb{R}^{N}} \cdot \mathrm{d} x$.

(3) $Q_{T}:=\mathbb{R}^{N} \times(0, T), \quad B_{R}:=\left\{x \in \mathbb{R}^{N} ;|x|<R\right\}$.

(4) When the weak derivatives $\nabla u, \nabla^{2} u$ and $\partial_{t} u$ are in $L^{p}\left(Q_{T}\right)$ for some $p \geq 1$, we say that $u \in W_{p}^{2,1}\left(Q_{T}\right)$, i.e.,

$$
\begin{aligned}
W_{p}^{2,1}\left(Q_{T}\right):=\left\{u \in L^{p}\left(0, T ; W^{2, p}\left(\mathbb{R}^{N}\right)\right) \cap W^{1, p}\left(0, T ; L^{p}\left(\mathbb{R}^{N}\right)\right)\right. & \\
& \left.\|u\|_{W_{p}^{2,1}\left(Q_{T}\right)}:=\|u\|_{L^{p}\left(Q_{T}\right)}+\|\nabla u\|_{L^{p}\left(Q_{T}\right)}+\left\|\nabla^{2} u\right\|_{L^{p}\left(Q_{T}\right)}+\left\|\partial_{t} u\right\|_{L^{p}\left(Q_{T}\right)}<\infty\right\}
\end{aligned}
$$

\section{Preliminary lemmas}

The following lemma gives us a version of Gagliardo-Nirenberg inequality. (See [33], Lem. 2.4. and Nakao [27])

Lemma 2.1. Let $N \geq 1, m \geq 1, a>2, u \in L^{q_{1}}\left(\mathbb{R}^{N}\right)$ with $q_{1} \geq 1$ and $u^{\frac{r+m-1}{2}} \in H^{1}\left(\mathbb{R}^{N}\right)$ with $r>0$. Let $q_{1} \in[1, r+m-1], q_{2} \in\left[\frac{r+m-1}{2}, \frac{a(r+m-1)}{2}\right]$ and

$$
\begin{cases}1 \leq q_{1} \leq q_{2} \leq \infty & \text { when } N=1 \\ 1 \leq q_{1} \leq q_{2}<\infty & \text { when } N=2 \\ 1 \leq q_{1} \leq q_{2} \leq \frac{(r+m-1) N}{N-2} & \text { when } N \geq 3\end{cases}
$$


Then, it holds that

$$
\|u\|_{L^{q_{2}}\left(\mathbf{R}^{N}\right)} \leq C^{\frac{2}{r+m-1}}\|u\|_{L^{q_{1}\left(\mathbf{R}^{N}\right)}}^{1-\Theta} \cdot\left\|\nabla u^{\frac{r+m-1}{2}}\right\|_{L^{2}\left(\mathbf{R}^{N}\right)}^{\frac{2 \Theta}{r+m-1}}
$$

with

$$
\Theta=\frac{r+m-1}{2} \cdot\left(\frac{1}{q_{1}}-\frac{1}{q_{2}}\right) \cdot\left(\frac{1}{N}-\frac{1}{2}+\frac{r+m-1}{2 q_{1}}\right)^{-1}
$$

where

and

$$
\begin{cases}C \text { depends only on } N \text { and } a & \text { when } q_{1} \geq \frac{r+m-1}{2}, \\ C=c_{0}^{\frac{1}{\beta}} \text { with } c_{0} \text { depending only on } N \text { and } a & \text { when } 1 \leq q_{1}<\frac{r+m-1}{2}\end{cases}
$$

$$
\beta:=\frac{q_{2}-\frac{r+m-1}{2}}{q_{2}-q_{1}}\left[\frac{2 q_{1}}{r+m-1}+\left(1-\frac{2 q_{1}}{r+m-1}\right) \frac{2 N}{N+2}\right] .
$$

The following inequalities are well known. (For instance, see Duoandikoetxea [9], p. 110 and Brezis [6], IX.12.) Lemma 2.2. Let $w \in W^{2, r}\left(\mathbb{R}^{N}\right)$. Then, the following inequalities hold:

$$
\begin{array}{cl}
\left\|\nabla^{2} w\right\|_{L^{r}\left(\mathbf{R}^{N}\right)} \leq C\left(\frac{r^{2}}{r-1}\right)^{2}\|\Delta w\|_{L^{r}\left(\mathbf{R}^{N}\right)} & \text { for } 1<r<\infty \\
\|w\|_{L^{\infty}\left(\mathbf{R}^{N}\right)} \leq \frac{2 r}{r-N}\|w\|_{W^{1, r}\left(\mathbf{R}^{N}\right)} & \text { for } r>N,
\end{array}
$$

where $C$ is a positive constant depending only on $N$.

We prepare a technical lemma which is used often when establishing the uniform bound of a solution $w_{k}$ for $(1)_{w}$ in ${ }^{w}(\mathrm{KS})$.

Lemma 2.3. Let $\eta=\eta(r)$ be as

$$
\eta(r):= \begin{cases}1 & \text { for } \quad r \geq 1 \\ \exp \left(1-\frac{1}{r}\right) & \text { for } 0 \leq r \leq 1 \\ 0 & \text { for } \quad r \leq 0\end{cases}
$$

We define a sequence $\eta_{\delta}(t)$ of cut-off functions by $\eta_{\delta}(t):=\eta\left(\frac{t}{\delta}\right)$. Then, it holds that

$$
\sup _{0<t<\infty} t^{-p} \eta_{\delta}(t) \leq \frac{p^{p}}{\delta^{p} e^{p-1}} \quad \text { and } \quad \sup _{0<t<\infty} t^{-p} \partial_{t} \eta_{\delta}(t) \leq \frac{(p+2)^{p+2}}{(\delta e)^{p+1}} \quad \text { for any } p \geq 1 \text {. }
$$

Proof of Lemma 2.3. For any $p \geq 1$, we have

$$
\sup _{0<t<\infty} t^{-p} \eta_{\delta}(t)=\sup _{0<t<\infty} \frac{e}{t^{p} e^{\frac{\delta}{t}}}=\frac{e}{\delta^{p}} \cdot \sup _{0<y<\infty} \frac{y^{p}}{e^{y}} \leq \frac{e}{\delta^{p}} \cdot \frac{p^{p}}{e^{p}}=\frac{p^{p}}{\delta^{p} e^{p-1}} .
$$

By the definition of $\eta$ and $\eta_{\delta}$, we see that

$$
\eta^{\prime}(r)= \begin{cases}0 & \text { for } \quad r \geq 1 \\ \frac{1}{r^{2}} \cdot \exp \left(1-\frac{1}{r}\right) & \text { for } \quad 0 \leq r \leq 1\end{cases}
$$


and

$$
\partial_{t} \eta_{\delta}(t)= \begin{cases}0 & \text { for } t \geq \delta \\ \frac{\delta}{t^{2}} \cdot \exp \left(1-\frac{\delta}{t}\right) & \text { for } \quad 0 \leq t \leq \delta\end{cases}
$$

From (2.11), we observe that

$$
\sup _{0<t<\infty} t^{-p} \partial_{t} \eta_{\delta}(t)=\sup _{0<t \leq \delta} \frac{\delta e}{t^{p+2} e^{\frac{\delta}{t}}}=\frac{\delta e}{\delta^{p+2}} \cdot \sup _{1 \leq y<\infty} \frac{y^{p+2}}{e^{y}} \leq \frac{\delta e}{\delta^{p+2}} \cdot \frac{(p+2)^{p+2}}{e^{p+2}}=\frac{(p+2)^{p+2}}{(\delta e)^{p+1}} .
$$

Thus, we complete the proof of Lemma 2.3 .

We present the crucial lemma which will play an important role when showing the asymptotic profile.

Lemma 2.4. Let $N \geq 1$ and $g$ belong to $L^{1}\left(\mathbb{R}^{N}\right)$ and assume that $\left\{w_{k}\right\}$ is a sequence of non-negative $L^{1}$-functions in $\mathbb{R}^{N}$ satisfying

$$
w_{k} \rightarrow g \quad \text { strongly in } L_{\text {loc }}^{1}\left(\mathbb{R}^{N}\right) .
$$

We also assume that for any fixed number $\delta>0$, there exist $f_{\delta} \in L^{1}\left(\mathbb{R}^{N}\right)$ and $k_{0} \in \mathbb{N}$ such that

$$
\int_{\mathbf{R}^{N}}\left[w_{k}-f_{\delta}\right]^{+} \mathrm{d} x<\frac{\delta}{6} \quad \text { for all } k>k_{0}
$$

where $[s]^{+}=\max (s, 0)$. Then, we have convergence:

$$
\left|\int_{\mathbf{R}^{N}}\left(w_{k}-g\right) \mathrm{d} x\right| \rightarrow 0 \quad \text { as } k \rightarrow \infty
$$

Proof of Lemma 2.4. Let $\delta$ be any fixed positive number and $\Omega$ be a domain in $\mathbb{R}^{N}$. Then, $\Omega$ can be written as the union

$$
\Omega=\Omega_{1} \cup \Omega_{2},
$$

where

$$
\Omega_{1}:=\left\{\Omega \subset \mathbb{R}^{N} ; w_{k}(x)-f_{\delta}(x) \geq 0\right\} \quad \text { and } \quad \Omega_{2}:=\left\{\Omega \subset \mathbb{R}^{N} ; w_{k}(x)-f_{\delta}(x)<0\right\} .
$$

Therefore, it holds that

$$
\begin{aligned}
\left|\int_{\Omega} w_{k}-f_{\delta} \mathrm{d} x\right| & \leq \int_{\Omega_{1}}\left|w_{k}-f_{\delta}\right| \mathrm{d} x+\int_{\Omega_{2}}\left|w_{k}-f_{\delta}\right| \mathrm{d} x \\
& =\int_{\Omega_{1}}\left(w_{k}-f_{\delta}\right) \mathrm{d} x+\int_{\Omega_{2}}\left(f_{\delta}-w_{k}\right) \mathrm{d} x \\
& =\int_{\mathbb{R}^{N}}\left[w_{k}-f_{\delta}\right]^{+} \mathrm{d} x+\int_{\Omega}\left|f_{\delta}\right| \mathrm{d} x .
\end{aligned}
$$

On the other hand, since $g, f_{\delta} \in L^{1}\left(\mathbb{R}^{N}\right)$, there exists a domain $K_{\delta} \subset \mathbb{R}^{N}$ depending on $\delta$ such that

$$
\int_{\mathbf{R}^{N} \backslash K_{\delta}}|g| \mathrm{d} x \leq \frac{\delta}{6} \quad \text { and } \quad \int_{\mathbf{R}^{N} \backslash K_{\delta}}\left|f_{\delta}\right| \mathrm{d} x \leq \frac{\delta}{6} .
$$


Taking $\Omega$ by $\Omega=\mathbb{R}^{N} \backslash K_{\delta}$ in (2.15), from (2.13) and (2.16), we observe that for any fixed number $\delta>0$, there exists $K_{\delta} \subset \mathbb{R}^{N}$ and $k_{0} \in \mathbb{N}$ such that

$$
\begin{array}{rlrl}
\left|\int_{\mathbb{R}^{N} \backslash K_{\delta}}\left(w_{k}-f_{\delta}\right) \mathrm{d} x\right| & \leq \int_{\mathbf{R}^{N}}\left[w_{k}-f_{\delta}\right]^{+} \mathrm{d} x+\int_{\mathbf{R}^{N} \backslash K_{\delta}}\left|f_{\delta}\right| \mathrm{d} x \\
& \leq \frac{\delta}{3} & \text { for all } k>k_{0} .
\end{array}
$$

Consequently, by $(2.12),(2.16)$ and $(2.17)$, we see that for any fixed number $\delta>0$, there exists $\tilde{k}_{0}\left(\geq k_{0}\right) \in \mathbb{N}$ such that

$$
\begin{aligned}
\left|\int_{\mathbf{R}^{N}}\left(w_{k}(x)-g(x)\right) \mathrm{d} x\right| & \leq\left|\int_{K_{\delta}}\left(w_{k}(x)-g(x)\right) \mathrm{d} x\right|+\left|\int_{\mathbb{R}^{N} \backslash K_{\delta}}\left(w_{k}(x)-g(x)\right) \mathrm{d} x\right| \\
& \leq\left\|w_{k}-g\right\|_{L^{1}\left(K_{\delta}\right)}+\left|\int_{\mathbb{R}^{N} \backslash K_{\delta}}\left(w_{k}(x)-f_{\delta}(x)\right) \mathrm{d} x\right|+\int_{\mathbf{R}^{N} \backslash K_{\delta}}\left|f_{\delta}(x)\right|+|g(x)| \mathrm{d} x \\
& \leq \frac{\delta}{3}+\frac{\delta}{3}+\frac{\delta}{6}+\frac{\delta}{6}=\delta
\end{aligned}
$$

for any $k>\tilde{k}_{0}$. We thus conclude (2.14) and complete the proof of Lemma 2.4.

\section{Approximated problem}

The first equation of (KS) is a quasi-linear parabolic equation of degenerate type. Therefore, we can not expect the problem $(\mathrm{KS})$ to have a classical solution at the point where the first solution $u$ vanishes. In order to justify all the formal arguments, we need to introduce the following approximated equation of (KS):

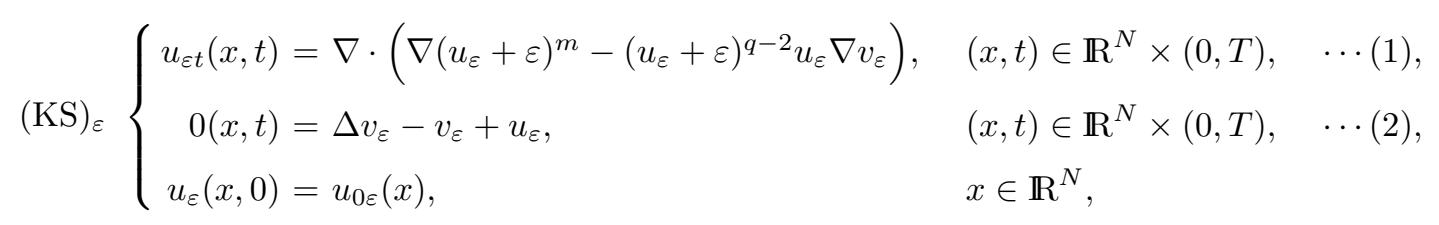

where $q>1$ and $\varepsilon$ is a positive parameter and $u_{0 \varepsilon}$ is an approximation for the initial data $u_{0}$ such that
(A.1): $0 \leq u_{0 \varepsilon} \in L^{1} \cap W^{2, p}\left(\mathbb{R}^{N}\right) \quad$ for all $\left\{\begin{array}{l}p \in\left[\frac{N}{N-1}, N+3\right], \\ p \in[2,3],\end{array}\right.$ for all $\varepsilon \in(0,1]$,
(A.2): $\left\|u_{0 \varepsilon}\right\|_{L^{p}} \leq\left\|u_{0}\right\|_{L^{p}} \quad$ for all $p \in[1, \infty], \quad$ for all $\varepsilon \in(0,1]$,
(A.3): $\left\|\nabla u_{0 \varepsilon}\right\|_{L^{2}} \leq\left\|\nabla u_{0}\right\|_{L^{2}} \quad$ for all $\varepsilon \in(0,1]$,
(A.4): $u_{0 \varepsilon} \rightarrow u_{0} \quad$ for some $p \in[1, \infty), \quad$ as $\varepsilon \rightarrow 0$.

We call $\left(u_{\varepsilon}, v_{\varepsilon}\right)$ a strong solution of $(\mathrm{KS})_{\varepsilon}$ if it belongs to $W_{p}^{2,1} \times W_{p}^{2,1}\left(Q_{T}\right)$ for some $p \geq 1$ and the equations $(1),(2)$ in $(\mathrm{KS})_{\varepsilon}$ are satisfied almost everywhere.

For the strong solution, we consider the space $\mathbf{W}\left(Q_{T}\right)$ defined by

$$
\begin{aligned}
\mathbf{W}\left(Q_{T}\right) & :=\mathbf{W}_{1}\left(Q_{T}\right) \times \mathbf{W}_{2}\left(Q_{T}\right) \\
& := \begin{cases}\left(W_{\frac{N}{N-1}}^{2,1} \bigcap W_{N+3}^{2,1}\left(Q_{T}\right)\right) \times W_{N+2}^{2,1}\left(Q_{T}\right) & \text { for } N \geq 2 \\
W_{3}^{2,1}\left(Q_{T}\right) \times W_{3}^{2,1}\left(Q_{T}\right) & \text { for } N=1 .\end{cases}
\end{aligned}
$$


In [30-32], the following proposition concerning the existence of the strong solution was proved:

Proposition 3.1 (time local existence,[30-32]). Let $N \geq 1, m>1$. Suppose that (A.1) is satisfied. Then, there exists a number $T_{1}=T_{1}\left(\varepsilon,\left\|u_{0 \varepsilon}\right\|_{W^{2, N+2}\left(\mathbf{R}^{N}\right)}, m, N\right)>0$ such that $(\mathrm{KS})_{\varepsilon}$ has the unique non-negative strong solution $\left(u_{\varepsilon}, v_{\varepsilon}\right)$ belonging to $\mathbf{W}\left(Q_{T_{1}}\right)$.

Proposition 3.2 (extension criterion,[30-32]). Let the same assumption as that in Proposition 3.1 hold and let $T>0$. Suppose that $\left(u_{\varepsilon}, v_{\varepsilon}\right)$ is a strong solution of $(\mathrm{KS})_{\varepsilon}$ in the class $\mathbf{W}\left(Q_{T}\right)$. If it holds that

$$
\sup _{0<t<T}\left\|u_{\varepsilon}(t)\right\|_{L^{\infty}\left(\mathbf{R}^{N}\right)}<\infty
$$

then there is $T^{\prime}>T$ such that $\left(u_{\varepsilon}, v_{\varepsilon}\right)$ can be a strong solution of $(\mathrm{KS})_{\varepsilon}$ in $\mathbf{W}\left(Q_{T^{\prime}}\right)$.

\section{Proof of Theorem 1.1}

For the rigorous proof, we multiply (1) in $(\mathrm{KS})_{\varepsilon}$ by $\left(u_{\varepsilon}+\varepsilon\right)^{r-1}$. For the sake of simplicity, we multiply $u_{\varepsilon t}=\nabla \cdot\left(\nabla\left(u_{\varepsilon}+\varepsilon\right)^{m}-\nabla u_{\varepsilon}^{q-1} \nabla v_{\varepsilon}\right)$ by $u_{\varepsilon}^{r-1}$, where $r>1$, and integrate it over $\mathbb{R}^{N}$. Then, we have

$$
\begin{aligned}
\frac{\mathrm{d}}{\mathrm{d} t}\left\|u_{\varepsilon}\right\|_{L^{r}}^{r} & \leq-m r(r-1) \int u_{\varepsilon}^{r+m-3}\left|\nabla u_{\varepsilon}\right|^{2} \mathrm{~d} x+r(r-1) \int u_{\varepsilon}^{q-1} \cdot \nabla v_{\varepsilon} \cdot u_{\varepsilon}^{r-2} \nabla u_{\varepsilon} \mathrm{d} x \\
& =-\frac{4 m r(r-1)}{(r+m-1)^{2}}\left\|\nabla u_{\varepsilon}^{\frac{r+m-1}{2}}\right\|_{L^{2}}^{2}+\frac{r(r-1)}{r+q-2} \int \nabla u_{\varepsilon}^{r+q-2} \cdot \nabla v_{\varepsilon} \mathrm{d} x \\
& =-\frac{4 m r(r-1)}{(r+m-1)^{2}}\left\|\nabla u_{\varepsilon}^{\frac{r+m-1}{2}}\right\|_{L^{2}}^{2}-\frac{r(r-1)}{r+q-2} \int u_{\varepsilon}^{r+q-2} \cdot \Delta v_{\varepsilon} \mathrm{d} x \\
& \leq-\frac{4 m r(r-1)}{(r+m-1)^{2}}\left\|\nabla u_{\varepsilon}^{\frac{r+m-1}{2}}\right\|_{L^{2}}^{2}+\frac{r(r-1)}{r+q-2}\left\|u_{\varepsilon}\right\|_{L^{r+q-1}}^{r+q-1} \quad \text { for all } r \in(1, \infty) .
\end{aligned}
$$

By taking $q_{1}=\frac{N(q-m)}{2}, q_{2}=r+q-1, a=2+\frac{2}{N}$ in Lemma 2.1, we have

$$
\left\|u_{\varepsilon}\right\|_{L^{r+q-1}}^{r+q-1} \leq c_{0}^{\frac{2(N+2)}{N} \cdot \frac{r+q-1}{r+m-1}}\left\|u_{\varepsilon}\right\|_{L^{\frac{N(q-m)}{2}}}^{q-m} \cdot\left\|\nabla u_{\varepsilon}^{\frac{r+m-1}{2}}\right\|_{L^{2}}^{2} \quad \text { for } r \geq \frac{N(q-m)}{2}
$$

for some absolute constant $c_{0}$, where we used

$$
\frac{1}{\beta}=\frac{N+2}{N} \cdot \frac{r+m-1}{r-m+2 q-1} \leq \frac{N+2}{N}
$$

by $m \leq q-\frac{2}{N}<q$.

Combining (4.1) with (4.2), we obtain

$$
\frac{\mathrm{d}}{\mathrm{d} t}\left\|u_{\varepsilon}\right\|_{L^{r}}^{r} \leq\left[\frac{r(r-1)}{r+q-2} c_{0}^{\frac{2(N+2)}{N} \cdot \frac{r+q-1}{r+m-1}}\left\|u_{\varepsilon}\right\|_{L^{\frac{N(q-m)}{2}}}^{q-m}-\frac{4 m r(r-1)}{(r+m-1)^{2}}\right]\left\|\nabla u_{\varepsilon}^{\frac{r+m-1}{2}}\right\|_{L^{2}}^{2} .
$$

By the Hölder inequality, $\left\|u_{0}\right\|_{L^{\frac{N(q-m)}{2}}} \leq\left\|u_{0}\right\|_{L^{1}}^{\gamma}\left\|u_{0}\right\|_{L^{\ell}}^{1-\gamma}$ for some $\gamma=\gamma(m, q, N, \ell)$. Therefore, when we take $\left\|u_{0}\right\|_{L^{\ell}}$ is sufficiently small for any fixed $\ell \geq \frac{N(q-m)}{2}$, it holds that $\left\|u_{0}\right\|_{L} \frac{N(q-m)}{2}$ is small.

On the other hand, $\left\|u_{\varepsilon}(t)\right\|_{L \frac{N(q-m)}{2}} \in C([0, T])$. Therefore, using this continuity and (4.3) with $r=$ $\frac{N(q-m)}{2}$, we find that there exists a short interval $\left[0, t_{1}\right]$ such that $\frac{\mathrm{d}}{\mathrm{d} t}\left\|u_{\varepsilon}(t)\right\|_{L^{r}} \leq 0$ for $t \in\left[0, t_{1}\right]$, and 
$\left\|u_{\varepsilon}(t)\right\|_{L} \frac{N(q-m)}{2} \leq\left\|u_{0}\right\|_{L} \frac{N(q-m)}{2}$ for $t \in\left[0, t_{1}\right]$. Since this implies that $\left\|u_{\varepsilon}\left(t_{1}\right)\right\|_{L} \frac{N(q-m)}{2} \leq\left\|u_{0}\right\|_{L} \frac{N(q-m)}{2}$, we can repeat this procedure. In consequence, we obtain

$$
\left\|u_{\varepsilon}(t)\right\|_{L} \frac{N(q-m)}{2} \leq\left\|u_{0}\right\|_{L} \frac{N(q-m)}{2} \quad \text { for } t \in[0, T]
$$

Substituting (4.4) into (4.3), we have

$$
\begin{array}{rlr}
\frac{\mathrm{d}}{\mathrm{d} t}\left\|u_{\varepsilon}\right\|_{L^{r}}^{r} & \leq\left(\frac{r(r-1)}{r+q-2} c_{0}^{\frac{2(N+2)}{N} \cdot \frac{r+q-1}{r+m-1}}\left\|u_{0}\right\|_{L}^{q-\frac{N(q-m)}{2}}-\frac{4 m r(r-1)}{(r+m-1)^{2}}\right)\left\|\nabla u_{\varepsilon}^{\frac{r+m-1}{2}}\right\|_{L^{2}}^{2} \\
& \leq-\frac{2 m r(r-1)}{(r+m-1)^{2}}\left\|\nabla u_{\varepsilon}^{\frac{r+m-1}{2}}\right\|_{L^{2}}^{2} & \\
& \leq 0 & \text { for } r \in\left[\frac{N(q-m)}{2}, \infty\right) .
\end{array}
$$

By applying Moser's iteration technique, we obtain

$$
\sup _{0<t<T}\left\|u_{\varepsilon}(t)\right\|_{L^{\infty}\left(\mathbb{R}^{N}\right)}<\infty
$$

(see [32], Lem. 15 or [33], Sect. 5). Combining (4.6) with Propositions 3.1 and 3.2, we prove the following lemma:

Lemma 4.1. Let $N \geq 1, m>1, q \geq m+\frac{N}{2}, \ell \geq \frac{N(q-m)}{2}(\geq 1), T>0$ and suppose that (A.1) is satisfied. Then, there exist an absolute constant $M$ and a positive number $\hat{\varepsilon}$ depending only on $M, N, m, \ell$ such that if $u_{0} \in L^{1} \cap L^{\ell}\left(\mathbb{R}^{N}\right)$ satisfies that

$$
\left\|u_{0}\right\|_{L^{1}\left(\mathbf{R}^{N}\right)}=M, \quad\left\|u_{0}\right\|_{L^{\ell}\left(\mathbf{R}^{N}\right)} \leq \hat{\varepsilon}
$$

then $(\mathrm{KS})_{\varepsilon}$ has the strong solution $\left(u_{\varepsilon}, v_{\varepsilon}\right)$ in the class obtained in $\mathbf{W}\left(Q_{T}\right)$ with the following property:

$$
\frac{\mathrm{d}}{\mathrm{d} t}\left\|u_{\varepsilon}\right\|_{L^{r}}^{r}+\frac{2 m r(r-1)}{(r+m-1)^{2}}\left\|\nabla u_{\varepsilon}^{\frac{r+m-1}{2}}\right\|_{L^{2}}^{2} \leq 0 \quad \text { for all } t \in(0, T) \text { and } r \in\left[\frac{N(q-m)}{2}, \infty\right) .
$$

From Lemma 4.1, we are going to show (1.6) in Theorem 1.1.

Lemma 2.1 with $a=3$ and (A.2) gives that

$$
\left\|u_{\varepsilon}\right\|_{L^{r}} \leq c^{\frac{1}{\beta_{1}} \cdot \frac{2}{r+m-1}}\left\|u_{0}\right\|_{L^{1}}^{1-\theta_{1}} \cdot\left\|\nabla u_{\varepsilon}^{\frac{r+m-1}{2}}\right\|_{L^{2}}^{\frac{2 \theta_{1}}{r+m-1}} \quad \text { for any } r \in[2, \infty)
$$

where $c$ depends only on $N$, and

$$
\begin{aligned}
& \beta_{1}:=\frac{N}{N+2} \frac{\left(r+m-2+\frac{2}{N}\right)(r-m+1)}{(r-1)(r+m-1)} \\
& \theta_{1}:=\frac{r+m-1}{2} \cdot\left(1-\frac{1}{r}\right) \cdot \frac{1}{\frac{1}{N}-\frac{1}{2}+\frac{r+m-1}{2}}
\end{aligned}
$$

Here and in what follows, $c$ denotes a general constant (not necessarily the same at different occurrences) but which depends only on $N$. 
Noting that

$$
\begin{array}{ll}
\frac{1}{\theta_{1}} \leq 2, \quad \frac{1-\theta_{1}}{\theta_{1}}(r+m-1) \leq \frac{N+2}{N} & \text { for } r \in[q, \infty), \\
\frac{1}{\beta_{1}} \leq \frac{2(N+2)}{N} & \text { for } r \in[3(m-1), \infty),
\end{array}
$$

we obtain

$$
\left\|u_{\varepsilon}\right\|_{L^{r}}^{\frac{r+m-1}{\theta_{1}}} \leq c\left\|u_{0}\right\|_{L^{1}}^{\frac{N+2}{N}} \cdot\left\|\nabla u_{\varepsilon}^{\frac{r+m-1}{2}}\right\|_{L^{2}}^{2} \quad \text { for any } r \in[\max \{q, 3(m-1)\}, \infty)
$$

By (4.9), we easily see that

$$
C_{m, r} \cdot\left\|u_{\varepsilon}\right\|_{L^{r}}^{r \cdot \lambda} \leq \frac{2 m r(r-1)}{(r+m-1)^{2}}\left\|\nabla u_{\varepsilon}^{\frac{r+m-1}{2}}\right\|_{L^{2}}^{2} \quad \text { for } m>1-\frac{2}{N}
$$

where

$$
\begin{aligned}
\lambda & :=\frac{r+m-1}{\theta_{1} \cdot r}=1+\frac{m-1+\frac{2}{N}}{r-1}>1, \\
C_{m, r} & :=\frac{2 m r(r-1)}{(r+m-1)^{2}} \cdot\left(c\left\|u_{0}\right\|_{L^{1}}\right)^{-\frac{N+2}{N}} .
\end{aligned}
$$

By combining (4.10) with Lemma 4.1,

$$
\frac{\mathrm{d}}{\mathrm{d} t}\left\|u_{\varepsilon}(t)\right\|_{L^{r}}^{r}+C_{m, r}\left\|u_{\varepsilon}(t)\right\|_{L^{r}}^{r \cdot \lambda} \leq 0 \quad \text { for } r \in[(N+2) q, \infty)
$$

Let us denote $\left\|u_{\varepsilon}(t)\right\|_{L^{r}}^{r}$ by $X(t)$. Then, (4.11) gives

$$
\frac{X(t)^{\prime}}{X(t)^{\lambda}}+C_{m, r}=\frac{1}{1-\lambda} \cdot\left(X(t)^{-\lambda+1}\right)^{\prime}+C_{m, r} \leq 0
$$

From (4.12), we obtain

$$
\begin{aligned}
X(t) & \leq \frac{1}{\left((\lambda-1) C_{m, r} \cdot t+X(0)^{-\lambda+1}\right)^{\frac{1}{\lambda-1}}} \\
& \leq \frac{1}{\min \left\{(\lambda-1) C_{m, r},\left\|u_{0 \varepsilon}\right\|_{L^{r}}^{r(-\lambda+1)}\right\}^{\frac{1}{\lambda-1}}} \cdot \frac{1}{(1+t)^{\frac{1}{\lambda-1}}} \\
& =\max \left\{\left((\lambda-1) C_{m, r}\right)^{-\frac{1}{\lambda-1}},\left\|u_{0 \varepsilon}\right\|_{L^{r}}^{r}\right\} \cdot(1+t)^{-\frac{1}{\lambda-1}} .
\end{aligned}
$$

This means that

$$
\begin{aligned}
\left\|u_{\varepsilon}(t)\right\|_{L^{r}} & \leq \max \left\{\left((\lambda-1) C_{m, r}\right)^{-\frac{1}{\lambda-1} \cdot \frac{1}{r}},\left\|u_{0 \varepsilon}\right\|_{L^{r}}\right\} \cdot(1+t)^{-\frac{1}{\lambda-1} \cdot \frac{1}{r}} \\
& \leq \tilde{C}_{0, r}(1+t)^{-\frac{N}{(m-1) N+2} \cdot\left(1-\frac{1}{r}\right)}
\end{aligned}
$$


where

$$
\tilde{C}_{0, r}:=\max \left\{\left[\frac{(r+m-1)^{2}}{r} \cdot \frac{1}{2 m\left(m-1+\frac{2}{N}\right)} \cdot\left(c\left\|u_{0}\right\|_{L^{1}}\right)^{\frac{N+2}{N}}\right]^{\frac{N}{(m-1) N+2} \cdot\left(1-\frac{1}{r}\right)},\left\|u_{0}\right\|_{L^{r}}\right\}
$$

We thus establish the decay estimate for $r \in[(N+2) q, \infty)$. On the other hand, by the Hölder inequality and the mass conservation law,

$$
\left\|u_{\varepsilon}\right\|_{L^{p}} \leq\left\|u_{0}\right\|_{L^{1}}^{1-\frac{p-1}{p} \cdot \frac{r}{r-1}}\left\|u_{\varepsilon}\right\|_{L^{r}}^{\frac{p-1}{p} \cdot \frac{r}{r-1}} \quad \text { for } p \in[1, r] .
$$

Therefore, we have the $L^{p}$-decay estimates for all $p \in[1, \infty)$ as follows:

$$
\left\|u_{\varepsilon}(t)\right\|_{L^{p}} \leq C_{0, p} \cdot(1+t)^{\frac{N}{(m-1) N+2} \cdot\left(1-\frac{1}{p}\right)} \quad \text { for } p \in[1, \infty)
$$

where

$$
C_{0, p}:=\left\|u_{0}\right\|_{L^{1}}^{1-\frac{p-1}{p} \cdot \frac{r}{r-1}} \cdot \tilde{C}_{0, r}^{\frac{p-1}{p} \cdot \frac{r}{r-1}} .
$$

In addition, a solution $v_{\varepsilon}$ of the second equation in $(\mathrm{KS})_{\varepsilon}$ can be expressed by the Bessel potential. Therefore, we obtain the the same decay estimate as (4.16) for $v_{\varepsilon}$.

Furthermore, by the similar argument to that in Section 5 in [32], we can prove that there exists a subsequence $\left\{u_{\varepsilon_{n}}\right\}$ such that

$$
\begin{array}{ccl}
u_{\varepsilon_{n}} \rightarrow u & \text { strongly } & \text { in } C\left((0, \infty) ; L_{l o c}^{p}\left(\mathbb{R}^{N}\right)\right), \\
\nabla u_{\varepsilon_{n}}^{m} \rightarrow \nabla u^{m} & \text { weakly star } & \text { in } L^{\infty}\left(0, \infty ; L^{2}\left(\mathbb{R}^{N}\right)\right), \\
v_{\varepsilon_{n}} \rightarrow v & \text { weakly star } & \text { in } L^{\infty}\left(0, \infty ; L^{s}\left(\mathbb{R}^{N}\right)\right), \\
\nabla v_{\varepsilon_{n}} \rightarrow \nabla v & \text { weakly star } & \text { in } L^{\infty}\left(0, \infty ; L^{s}\left(\mathbb{R}^{N}\right)\right), \\
\Delta v_{\varepsilon_{n}} \rightarrow \Delta v & \text { weakly star } & \text { in } L^{\infty}\left(0, \infty ; L^{s}\left(\mathbb{R}^{N}\right)\right)
\end{array}
$$

for any $p \in[1, \infty)$ and any $s \in(1, \infty]$. Hence, by the standard convergence argument, we prove the existence of a weak solution $(u, v)$ for (KS). Moreover, by the lower semi-continuity of the norm for $p \in(1, \infty)$ and Fatou lemma for $p=1$, we obtain the decay estimate (1.6) in Theorem 1.1. Thus, we complete the proof of Theorem 1.1.

\section{Proof of Theorem 1.2}

We set $\left(w_{k}, v_{k}\right)$ by

$$
w_{k}(x, t):=k^{N} u\left(k x, k^{N(m-1)+2} t\right) \quad \text { and } \quad z_{k}(x, t):=k^{N} v\left(k x, k^{N(m-1)+2} t\right) \quad \text { for } k>1 .
$$

Then, the above $\left(w_{k}, v_{k}\right)$ becomes a non-negative weak solution of the following problem:

$$
{ }^{w}(\mathrm{KS})\left\{\begin{aligned}
w_{k t}(x, t) & =\nabla \cdot\left(\nabla\left(w_{k}\right)^{m}-k^{-N(q-m)}\left(w_{k}\right)^{q-1} \cdot \nabla z_{k}\right), & & (x, t) \in \mathbb{R}^{N} \times(0, \infty), \\
0 & =\Delta z_{k}-k^{2} z_{k}+k^{2} w_{k}, & & (x, t) \in \mathbb{R}^{N} \times(0, \infty), \quad \cdots(2)_{w} \\
w_{k}(x, 0) & =k^{N} u_{0}(k x), & & x \in \mathbb{R}^{N},
\end{aligned}\right.
$$

where $N \geq 1, m>1, q \geq \max \left\{m+\frac{2}{N}, 2\right\}$. 


\subsection{A priori estimate for $w_{k}$}

By (4.15)-(4.16) in the proof of Theorem 1.1, we see that there exists a constant $C_{0, p}=C_{0, p}\left(m, q, N, p,\left\|u_{0}\right\|_{L^{1}}\right.$, $\left.\left\|u_{0}\right\|_{L^{p}},\left\|u_{0}\right\|_{L^{(N+2) q}}\right)$ independently of $k$ such that

$$
\left\|w_{k}(t)\right\|_{L^{p}}=k^{N\left(1-\frac{1}{p}\right)}\left\|u\left(k^{N(m-1)+2} t\right)\right\|_{L^{p}} \leq C_{0, p} t^{-\frac{N}{N(m-1)+2} \cdot\left(1-\frac{1}{p}\right)} \quad \text { for } t>0 \text { and } p \in[1, \infty) .
$$

Moreover, we obtain the $L^{\infty}\left(\delta, T ; L^{\infty}\left(\mathbb{R}^{N}\right)\right.$ )-estimate (for any $\delta>0$ ) for $w_{k}$ by Moser's iteration technique. To this end, we prepare the $L^{\infty}\left(\delta, T ; L^{p}\left(\mathbb{R}^{N}\right)\right)$-estimate for $w_{k}$ using (5.2).

Lemma 5.1. Let $\delta>0, p \geq 1$ and let $N \geq 1, m>1, q \geq \max \left\{m+\frac{2}{N}, 2\right\}$. Suppose that $\left(w_{k}, z_{k}\right)$ is a weak solution of ${ }^{w}(\mathrm{KS})$. We assume that $w_{k}$ satisfies (5.2). Then, there exist positive numbers $R_{\delta, p}, Q_{\delta, p}$ depending on $\delta, p, m, q, N,\left\|u_{0}\right\|_{L^{1} \cap L^{p} \cap L^{(N+2) q}\left(\mathbf{R}^{N}\right)}$ but not on $k$ such that

$$
\sup _{0<t<\infty}\left(\left\|w_{k}\right\|_{L^{p}\left(\mathbf{R}^{N}\right)}^{p} \eta_{\delta}(t)\right) \leq R_{\delta, p} \quad \text { and } \quad \sup _{0<t<\infty}\left(\left\|w_{k}\right\|_{L^{p}\left(\mathbf{R}^{N}\right)}^{p} \partial_{t} \eta_{\delta}(t)\right) \leq Q_{\delta, p}
$$

where $\eta_{\delta}(t)$ is a sequence of cut-off functions defined by $\eta_{\delta}(t):=\eta\left(\frac{t}{\delta}\right)$ with $\eta$ introduced in Lemma 2.3.

Proof of Lemma 5.1. From (5.2) and Lemma 2.3, we see that

$$
\begin{aligned}
\sup _{0<t<\infty}\left(\left\|w_{k}\right\|_{L^{p}}^{p} \eta_{\delta}(t)\right) & \leq \sup _{0<t<\delta}\left(\left\|w_{k}\right\|_{L^{p}}^{p} \eta_{\delta}(t)\right)+\sup _{\delta<t<\infty}\left(\left\|w_{k}\right\|_{L^{p}}^{p} \eta_{\delta}(t)\right) \\
& \leq\left(C_{0, p}\right)^{p} \sup _{0<t<\delta}\left(t^{-\frac{N}{N(m-1)+2} \cdot(p-1)} \eta_{\delta}(t)\right)+\left(C_{0, p}\right)^{p} \sup _{\delta<t<\infty} t^{-\frac{N}{N(m-1)+2} \cdot(p-1)} \\
& \leq\left(C_{0, p}\right)^{p} \frac{\mu^{\mu}}{\delta^{\mu} e^{\mu-1}}+\left(C_{0, p}\right)^{p} \delta^{-\frac{N}{N(m-1)+2} \cdot(p-1)}=: R_{\delta, p} \\
\sup _{0<t<\infty}\left(\left\|w_{k}\right\|_{L^{p}}^{p} \partial_{t} \eta_{\delta}(t)\right) & =\sup _{0<t<\delta}\left(\left\|w_{k}\right\|_{L^{p}}^{p} \partial_{t} \eta_{\delta}(t)\right) \\
& \leq\left(C_{0, p}\right)^{p} \sup _{0<t<\delta}\left(t^{-\frac{N}{N(m-1)+2} \cdot(p-1)} \partial_{t} \eta_{\delta}(t)\right) \leq\left(C_{0, p}\right)^{p} \frac{(\mu+2)^{\mu+2}}{(\delta e)^{\mu+1}} \\
& =\left(C_{0, p}\right)^{p} \frac{(\mu+2)^{\mu+2}}{(\delta e)^{\mu+1}}=: Q_{\delta, p},
\end{aligned}
$$

where $\mu:=\frac{N}{N(m-1)+2} \cdot(p-1)$.

From $(2)_{w}$ in ${ }^{w}(\mathrm{KS})$ and (5.2), we see by the standard argument that

$$
\begin{aligned}
& \sup _{\delta<t<\infty}\left\|z_{k}(t)\right\|_{L^{p}} \leq C_{0, p} \delta^{-\frac{\mu}{p}}, \sup _{\delta<t<\infty}\left\|\Delta z_{k}(t)\right\|_{L^{p}} \leq 2 k^{2} C_{0, p} \delta^{-\frac{\mu}{p}}, \\
& \sup _{\delta<t<\infty}\left\|\nabla z_{k}(t)\right\|_{L^{p}} \leq k\left(p+N^{2}-1\right)^{\frac{1}{2}} C_{0, p} \delta^{-\frac{\mu}{p}} \quad \text { for any } \delta>0 \text { and } p \in[2, \infty),
\end{aligned}
$$

where $C_{0, p}$ is the constant in $(5.2)$ and $\mu:=\frac{N}{N(m-1)+2} \cdot(p-1)$. Moreover, by (2.6) and (2.7) in Lemma 2.2, we see that

$$
\begin{aligned}
\left\|\nabla z_{k}(t)\right\|_{L^{\infty}} & \leq \frac{2 p}{p-N} \cdot\left(\left\|\nabla z_{k}(t)\right\|_{L^{p}}+\left\|\nabla^{2} z_{k}(t)\right\|_{L^{p}}\right) \\
& \leq \frac{2 p}{p-N} \cdot c\left(\frac{p^{2}}{p-1}\right)^{2}\left(\left\|\nabla z_{k}(t)\right\|_{L^{p}}+\left\|\Delta z_{k}(t)\right\|_{L^{p}}\right) \\
& \leq \frac{2 p}{p-N} \cdot c\left(\frac{p^{2}}{p-1}\right)^{2} \cdot\left(p+N^{2}+1\right) C_{0, p} \delta^{-\frac{\mu}{p}} \cdot k^{2} \quad \text { for } p \in(N, \infty) .
\end{aligned}
$$


Therefore, taking $p=N+1$, we have

$$
\sup _{\delta<t<\infty}\left\|\nabla z_{k}(t)\right\|_{L^{\infty}} \leq c \cdot C_{0, N+1} \delta^{-\frac{\mu}{N+1}} \cdot k^{2}=: C_{\nabla z} \cdot k^{2} \quad \text { for any } \delta>0
$$

Using (5.5), (5.6) and Lemma 5.1, we establish the $L^{\infty}$-estimate for $w_{k}$ in the following lemma.

Lemma 5.2. Let the same assumption as that in Lemma 5.1 hold and let $T>0$. Then, there exists a positive number $W_{\delta, T}$ depending on $\delta, m, q, N,\left\|u_{0}\right\|_{L^{1} \cap L^{(N+2) q}\left(\mathbf{R}^{N}\right)}, T$ but not on $k$ such that

$$
\sup _{\delta<t<T}\left\|w_{k}(t)\right\|_{L^{\infty}\left(\mathbf{R}^{N}\right)} \leq W_{\delta, T} \quad \text { for all } \delta>0
$$

Proof of Lemma 5.2. We follow the argument employed in [33], Proof of Lemma 10. (For the sake of simplicity, we perform only the formal calculation.)

We multiply (1) in ${ }^{w}(\mathrm{KS})$ by $\left(w_{k}\right)^{p-1} \eta_{\delta}(t)$ and integrate it over $\mathbb{R}^{N}$, where $p>1$ and $\eta_{\delta}$ is the function defined in Lemma 5.1. Then, by $q \geq m+\frac{2}{N},(5.6)$ and Young inequality, we have

$$
\begin{aligned}
\frac{1}{p} \cdot \frac{\mathrm{d}}{\mathrm{d} t}\left(\left\|w_{k}\right\|_{L^{p}}^{p} \eta_{\delta}(t)\right) \leq & -\frac{4 m(p-1)}{(p+m-1)^{2}}\left\|\nabla w_{k}^{\frac{p+m-1}{2}}\right\|_{L^{2}}^{2} \eta_{\delta}(t) \\
& +\frac{1}{p}\left\|w_{k}\right\|_{L^{p}}^{p} \partial_{t} \eta_{\delta}(t)+k^{-N(q-m)}(p-1) \int\left(w_{k}\right)^{q-1} \nabla z_{k} \cdot\left(w_{k}\right)^{p-2} \nabla w_{k} \cdot \eta_{\delta}(t) \mathrm{d} x \\
\leq & -\frac{2 m(p-1)}{(p+m-1)^{2}}\left\|\nabla w_{k}^{\frac{p+m-1}{2}}\right\|_{L^{2}}^{2} \eta_{\delta}(t) \\
& +\frac{1}{p t^{2}}\left\|w_{k}\right\|_{L^{p}}^{p} \eta_{\delta}(t)+\frac{p-1}{2 m} \cdot k^{-2 N(q-m)+2}\left(C_{\nabla z}\right)^{2}\left\|w_{k}\right\|_{L^{p+2 q-3-m}}^{p+2 q-3-m} \eta_{\delta}(t) \\
\leq & -\frac{2 m(p-1)}{(p+m-1)^{2}}\left\|\nabla w_{k}^{\frac{p+m-1}{2}}\right\|_{L^{2}}^{2} \eta_{\delta}(t) \\
& +\frac{1}{p t^{2}}\left\|w_{k}\right\|_{L^{p}}^{p} \eta_{\delta}(t)+\frac{p-1}{2 m}\left(C_{\nabla z}\right)^{2}\left\|w_{k}(t)\right\|_{L^{1}(q-2)-1}^{\frac{m+2(q-1}{p+2}}\left\|w_{k}(t)\right\|_{L^{p+2(q-2)}}^{(p+2(q-2)) \cdot \frac{p-m+2(q-2)}{p+2(q-2)-1}} \eta_{\delta}(t) \\
\leq & -\frac{2 m(p-1)}{(p+m-1)^{2}}\left\|\nabla w_{k}^{\frac{p+m-1}{2}}\right\|_{L^{2}}^{2} \eta_{\delta}(t) \\
& +\frac{1}{p t^{2}}\left\|w_{k}\right\|_{L^{p}}^{p} \eta_{\delta}(t)+\left(\frac{1}{2 m}\left(C_{\nabla z}\right)^{2}\left\|w_{k}(0)\right\|_{L^{1}}^{\frac{m-2(q-2)-1}{p+2}}\right)^{\frac{p+2(q-2)-1}{m-1}} \\
& +p^{2}\left\|w_{k}(t)\right\|_{L^{p+2(q-2)}}^{p+2} \eta_{\delta}(t)
\end{aligned}
$$

for all $p \in[2 m-2 q+3, \infty)$.

Using Lemma 2.1, we see that there exists a positive number $p_{0}$ depending only on $m, q, N$ such that

$$
p^{2}\left\|w_{k}\right\|_{L^{p+2(q-2)}}^{p+2(q-2)} \leq \frac{m(q-1)}{(q+m-1)^{2}}\left\|\nabla\left(w_{k}\right)^{\frac{p+m-1}{2}}\right\|_{L^{2}}^{2}+1+(p+m-1)^{c} c\left\|w_{k}\right\|_{L^{\frac{p}{4}}}^{p} \eta_{\delta}^{4}(t)
$$

and

$$
\begin{aligned}
\frac{1}{p t^{2}}\left\|w_{k}\right\|_{L^{p}}^{p} \leq & \frac{m(p-1)}{(p+m-1)^{2}}\left\|\nabla\left(w_{k}\right)^{\frac{p+m-1}{2}}\right\|_{L^{2}}^{2}+1 \\
& +(p+m-1)^{c} c \cdot\left(\frac{1}{t^{2}}\right)^{(3 N+1)^{2}} \eta_{\delta}(t)^{3(3 N-1)(N+1)}\left\|w_{k}\right\|_{L^{\frac{p}{4}}}^{p} \eta_{\delta}^{4} \\
\leq & \frac{m(p-1)}{(p+m-1)^{2}}\left\|\nabla\left(w_{k}\right)^{\frac{p+m-1}{2}}\right\|_{L^{2}}^{2}+1+(p+m-1)^{c} c\left\|w_{k}\right\|_{L^{\frac{p}{4}}}^{p} \eta_{\delta}^{4}
\end{aligned}
$$

for all $p \in\left[p_{0}, \infty\right)$. 
Substituting (5.9) and (5.10) into (5.8) and integrating it from 0 to $t$, we have

$$
\begin{aligned}
\sup _{0<t<T}\left\|w_{k} \eta_{\delta}(t)^{\frac{1}{p}}\right\|_{L^{p}}^{p} \leq & \left(\frac{1}{2 m}\left(C_{\nabla z}\right)^{2}\left\|u_{0}\right\|_{L^{1}}^{\frac{m-1}{p+(q-2)-1}}\right)^{\frac{p+2(q-2)-1}{m-1}} T+2 p T \\
& +p T(p+m-1)^{c} c \sup _{0<t<T}\left\|w_{k} \eta_{\delta}(t)^{\frac{4}{p}}\right\|_{L^{\frac{p}{4}}}^{p}
\end{aligned}
$$

for all $p \in\left[p_{0}, \infty\right)$. Applying the Moser iteration technique to (5.11), we have

$$
\begin{aligned}
\sup _{0<t<T}\left\|w_{k} \eta_{\delta}(t)^{\frac{1}{p^{p}}}\right\|_{L^{4 p}} & \leq 4^{C_{1} p_{0} 4^{2-p_{0}}} C_{1}^{4^{2-p_{0}}} \max \left\{\left(\frac{1}{2 m}\left(C_{\nabla v}\right)^{2}\right)^{\frac{1}{m-1}}, 1, R_{\delta, 4^{p_{0}}}\right\} \\
& <\infty
\end{aligned}
$$

where $C_{1}$ is a constant depending only on $\delta, m, q, N, T,\left\|u_{0}\right\|_{L^{1}},\left\|u_{0}\right\|_{L^{N+1}}$. Consequently, by letting $p$ tend to $\infty$, we see that $w_{k} \in L^{\infty}\left(\delta, \infty ; L^{\infty}\left(\mathbb{R}^{N}\right)\right)$ and

$$
\sup _{\delta<t<T}\left\|w_{k}(t)\right\|_{L^{\infty}\left(\mathbb{R}^{N}\right)} \leq 4^{C_{1}\left(p_{0}\right) 4^{2-p_{0}}} C_{1}^{4^{2-p_{0}}} \max \left\{\left(\frac{1}{2 m}\left(C_{\nabla v}\right)^{2}\right)^{\frac{1}{m-1}}, 1, R_{\delta, 4^{p_{0}}}\right\}=: W_{\delta, T}
$$

for any fixed number $\delta>0$. Thus, the $L^{\infty}\left(\mathbb{R}^{N}\right)$-bound for $w_{k}$ is obtained, which complete the proof of Lemma 5.2.

Lemma 5.3. Let the same assumption as that in Lemma 5.1 hold and let $T>0$. Then, there exists a positive number $N_{\delta, T}$ depending on $\delta, m, q, N,\left\|u_{0}\right\|_{L^{1} \cap L^{4} \cap L^{(N+2) q}\left(\mathbf{R}^{N}\right)}, T$ but not on $k$ such that

$$
\int_{\delta}^{T}\left\|\partial_{t}\left(w_{k}\right)^{m}\right\|_{L^{2}\left(\mathbf{R}^{N}\right)}^{2} \mathrm{~d} t+\sup _{\delta<t<T}\left\|\nabla\left(w_{k}\right)^{m}(t)\right\|_{L^{2}\left(\mathbf{R}^{N}\right)}^{2} \leq N_{\delta, T} T
$$

Proof of Lemma 5.3. For the sake of simplicity, we perform only the formal calculation. We multiply (1) in ${ }^{w}(\mathrm{KS})$ by $\left(w_{k}\right)^{p-1} \eta_{\delta}(t)$ and integrate it over $\mathbb{R}^{N}$, where $p>1$ and $\eta_{\delta}$ is the function defined in Lemma 5.1. Then, similarly to (4.1), we have

$$
\begin{aligned}
\frac{\mathrm{d}}{\mathrm{d} t}\left(\left\|w_{k}\right\|_{\left.L^{p}\right)}^{p} \eta_{\delta}(t)\right)+\frac{4 m p(p-1)}{(p+m-1)^{2}} \| & \nabla w_{k}^{\frac{p+m-1}{2}}\left\|_{L^{2}}^{2} \eta_{\delta}(t) \leq\right\| w_{k} \|_{L^{p}}^{p} \partial_{t} \eta_{\delta}(t) \\
& +k^{-N(q-m)+2} \cdot \frac{p(p-1)}{p+q-2}\left\|w_{k}\right\|_{L^{p+q-1}}^{p+q-1} \eta_{\delta}(t) \quad \text { for all } p \in(1, \infty)
\end{aligned}
$$

Integrating (5.15) from 0 to $t$, from Lemma 5.1 and $q \geq m+\frac{2}{N}$, we have

$$
\begin{aligned}
\left\|w_{k}\right\|_{L^{p}}^{p} \eta_{\delta}(t)+\frac{4 m p(p-1)}{(p+m-1)^{2}} \int_{0}^{t}\left\|\nabla w_{k}^{\frac{p+m-1}{2}}\right\|_{L^{2}}^{2} \eta_{\delta}(s) \mathrm{d} s \leq & \int_{0}^{t}\left\|w_{k}\right\|_{L^{p}}^{p} \partial_{t} \eta_{\delta}(s) \mathrm{d} s \\
& +k^{-N(q-m)+2} \cdot \frac{p(p-1)}{p+q-2} \int_{0}^{t}\left\|w_{k}\right\|_{L^{p+q-1}}^{p+q-1} \eta_{\delta}(s) \mathrm{d} s \\
\leq & Q_{\delta, p} T+\frac{p(p-1)}{p+q-2} \cdot R_{\delta, p+q-1} T
\end{aligned}
$$

for all $p \in(1, \infty)$ and $t \in(0, T)$. 
Thus, we obtain

$$
\begin{aligned}
& \int_{0}^{T}\left\|\nabla w_{k}^{\frac{p+m-1}{2}}\right\|_{L^{2}}^{2} \eta_{\delta}(s) \mathrm{d} s \\
& \leq \frac{(p+m-1)^{2}}{4 m p(p-1)}\left(Q_{\delta, p}+\frac{p(p-1)}{p+q-2} \cdot R_{\delta, p+q-1}\right) T=: \quad L_{\delta, p} T \quad \text { for all } p \in(1, \infty) .
\end{aligned}
$$

By the similar argument, we find that there exists a positive number $\tilde{L}_{\delta, p}$ depending on $\delta, p, m, q, N$, $\left\|u_{0}\right\|_{L^{1}},\left\|u_{0}\right\|_{L^{p}}$ but not $k$ such that

$$
\int_{0}^{T}\left\|\nabla w_{k}^{\frac{p+m-1}{2}}\right\|_{L^{2}}^{2} \eta_{\delta}(t) \frac{1}{t^{2}} \mathrm{~d} t \leq \tilde{L}_{\delta, p} T \quad \text { for all } p \in(1, \infty)
$$

Next, we multiply (1) in ${ }^{w}(\mathrm{KS})$ by $\partial_{t}\left(w_{k}\right)^{m} \cdot \eta_{\delta}(t)$ and integrate it over $\mathbb{R}^{N}$. Noting that $\Delta z_{k}=k^{2}\left(z_{k}-w_{k}\right)$, from (5.4) and (5.6), we have

$$
\begin{aligned}
& \frac{4 m}{(m+1)^{2}} \cdot\left\|\partial_{t}\left(w_{k}\right)^{\frac{m+1}{2}}\right\|_{L^{2}}^{2} \eta_{\delta}(t)+\frac{1}{2} \cdot \frac{\mathrm{d}}{\mathrm{d} t}\left\|\nabla\left(w_{k}\right)^{m}\right\|_{L^{2}}^{2} \eta_{\delta}(t) \\
& =\frac{1}{2}\left\|\nabla\left(w_{k}\right)^{m}\right\|_{L^{2}}^{2} \partial_{t} \eta_{\delta}(t)-k^{-N(q-m)} \cdot \frac{2 m}{m+1} \int\left(w_{k}\right)^{\frac{m-1}{2}} \nabla\left(\left(w_{k}\right)^{q-1} \nabla z_{k}\right) \partial_{t}\left(w_{k}\right)^{\frac{m+1}{2}} \eta_{\delta}(t) \mathrm{d} x \\
& \leq \frac{1}{2}\left\|\nabla\left(w_{k}\right)^{m}\right\|_{L^{2}}^{2} \eta_{\delta}(t) \frac{\delta}{t^{2}}+\frac{m}{(m+1)^{2}}\left\|\partial_{t}\left(w_{k}\right)^{\frac{m+1}{2}}\right\|_{L^{2}}^{2} \eta_{\delta}(t) \\
& \quad+k^{-2 N(q-m)} m \int\left(w_{k}\right)^{m-1}\left|\nabla\left(\left(w_{k}\right)^{q-1} \nabla z_{k}\right)\right|^{2} \eta_{\delta}(t) \mathrm{d} x \\
& \leq \\
& \frac{1}{2}\left\|\nabla\left(w_{k}\right)^{m}\right\|_{L^{2}}^{2} \eta_{\delta}(t) \frac{\delta}{t^{2}}+\frac{m}{(m+1)^{2}}\left\|\partial_{t}\left(w_{k}\right)^{\frac{m+1}{2}}\right\|^{2} \eta_{\delta}(t) \\
& \quad+k^{-2 N(q-m)+4} \cdot 2 m\left(\frac{q-1}{q+\frac{m-3}{2}}\right)^{2} \cdot\left(C C_{\nabla z}\right)^{2}\left\|\nabla\left(w_{k}\right)^{q+\frac{m-3}{2}}\right\|_{L^{2}}^{2} \eta_{\delta}(t) \\
& \quad+k^{-2 N(q-m)+4} \cdot 2 m\left(2\left\|w_{k}\right\|_{L^{m+2 q-1}}^{m+2 q-1}+\left\|w_{k}\right\|_{L^{2(m+2 q-3)}}^{2(m+2 q-3)}+\left\|z_{k}\right\|_{L^{4}}^{4}\right) \eta_{\delta}(t) .
\end{aligned}
$$

Integrating (5.19) from 0 to $t$ and using (5.3), (5.4), (5.17), (5.18) and $q \geq \max \left\{m+\frac{2}{N}, 2\right\}$, we have

$$
\begin{aligned}
& \frac{3 m}{(m+1)^{2}} \cdot \int_{0}^{t}\left\|\partial_{t}\left(w_{k}\right)^{\frac{m+1}{2}}\right\|_{L^{2}}^{2} \eta_{\delta} \mathrm{d} t+\frac{1}{2}\left\|\nabla\left(w_{k}\right)^{m}\right\|_{L^{2}}^{2} \eta_{\delta}(t) \\
& \leq \frac{\delta}{2} \int_{0}^{t}\left\|\nabla\left(w_{k}\right)^{m}\right\|_{L^{2}}^{2} \eta_{\delta}(t) \frac{1}{t^{2}} \\
& \quad+k^{-2 N(q-m)+4} \cdot 2 m\left(\frac{q-1}{q+\frac{m-3}{2}}\right)^{2} \cdot\left(C_{\nabla z}\right)^{2} \int_{0}^{t}\left\|\nabla\left(w_{k}\right)^{q+\frac{m-3}{2}}\right\|_{L^{2}}^{2} \eta_{\delta}(t) \mathrm{d} t \\
& \quad+k^{-2 N(q-m)+4} \cdot 2 m \int_{0}^{t}\left(2\left\|w_{k}\right\|_{L^{m+2 q-1}}^{m+2 q-1}+\left\|w_{k}\right\|_{L^{m+2 q-3}}^{m+2 q-3}+\left\|z_{k}\right\|_{L^{4}}^{4}\right) \eta_{\delta}(t) \mathrm{d} t \\
& \leq \frac{\delta}{2} \tilde{L}_{\delta, m+1} T+2 m\left(\frac{q-1}{q+\frac{m-3}{2}}\right)^{2} \cdot\left(C_{\nabla z}\right)^{2} \cdot L_{\delta, 2 q-2} T \\
& \quad+2 m\left(2 R_{\delta, m+2 q-1}+R_{\delta, 2(m+2 q-3)}+\left(C_{0,4}\right)^{4} \delta^{-\frac{4 \mu}{p}}\right) T
\end{aligned}
$$


for $t \in(0, T)$ and $\mu=\frac{N}{N(m-1)+2} \cdot(p-1)$. Therefore, we find that

$$
\begin{aligned}
\sup _{\delta<t<T}\left\|\nabla\left(w_{k}\right)^{m}(t)\right\|_{L^{2}}^{2} \leq & \delta \tilde{L}_{\delta, m+1} T+2\left[2 m\left(\frac{q-1}{q+\frac{m-3}{2}}\right)^{2} \cdot\left(C_{\nabla z}\right)^{2} \cdot L_{\delta, 2 q-2}\right. \\
& \left.+2 m\left(2 R_{\delta, m+2 q-1}+R_{\delta, 2(m+2 q-3)}+\left(C_{0,4}\right)^{4} \delta^{-\frac{4 \mu}{p}}\right)\right] T \\
=: & N_{\delta}^{(1)} T .
\end{aligned}
$$

Moreover, it holds that

$$
\begin{aligned}
\int_{\delta}^{T}\left\|\partial_{t}\left(w_{k}\right)^{\frac{m+1}{2}}\right\|_{L^{2}}^{2} \mathrm{~d} t \leq & \frac{\delta(m+1)^{2}}{6 m} \tilde{L}_{\delta, m+1} T+\frac{(m+1)^{2}}{3 m}\left[2 m\left(\frac{q-1}{q+\frac{m-3}{2}}\right)^{2} \cdot\left(C_{\nabla z}\right)^{2} \cdot L_{\delta, 2 q-2}\right. \\
& \left.+2 m\left(2 R_{\delta, m+2 q-1}+R_{\delta, 2(m+2 q-3)}+\left(C_{0,4}\right)^{4} \delta^{-\frac{4 \mu}{p}}\right)\right] T \\
= & : N_{\delta}^{(2)} T
\end{aligned}
$$

From (5.20) and Lemma 5.2, we find that

$$
\begin{aligned}
\int_{\delta}^{T}\left\|\partial_{t}\left(w_{k}\right)^{m}\right\|_{L^{2}}^{2} \mathrm{~d} t & \leq\left(\frac{2 m}{m+1}\right)^{2}\left(W_{\delta, T}\right)^{m-1} \int_{\delta}^{T}\left\|\partial_{t}\left(w_{k}\right)^{\frac{m+1}{2}}\right\|_{L^{2}}^{2} \mathrm{~d} t \\
& \leq\left(\frac{2 m}{m+1}\right)^{2}\left(W_{\delta, T}\right)^{m-1} N_{\delta}^{(2)} T=: N_{\delta, T}^{(3)} T .
\end{aligned}
$$

Thus, we establish

$$
\int_{\delta}^{T}\left\|\partial_{t}\left(w_{k}\right)^{m}\right\|_{L^{2}}^{2} \mathrm{~d} t+\sup _{\delta<t<T}\left\|\nabla\left(w_{k}\right)^{m}(t)\right\|_{L^{2}}^{2} \leq\left(N_{\delta}^{(1)}+N_{\delta, T}^{(3)}\right) T=: N_{\delta, T} T
$$

for any fixed number $\delta>0$, which completes the proof of Lemma 5.3.

\subsection{Convergence}

From (5.2), we find that $\left\|w_{k}(t)\right\|_{L^{p}}$ is bounded on $[\delta, \infty)$ for any $\delta>0$. Therefore, we can extract a subsequence $\left\{w_{k_{n}}\right\}$ which converges in $L^{p}(1<p<\infty)$ such that

$$
w_{k_{n}} \rightarrow \quad U \quad \text { weakly } \quad \text { in } L^{p}\left(\delta, T ; L^{p}\left(\mathbb{R}^{N}\right)\right)
$$

for any $T>0$ and $\delta \in(0, T)$. Moreover, we see that there exists a subsequence, still denoted by $\left\{w_{k_{n}}\right\}$ such that

$$
\begin{aligned}
& w_{k_{n}} \quad \rightarrow U \quad \text { strongly } \quad \text { in } C\left((0, T) ; L_{l o c}^{p}\left(\mathbb{R}^{N}\right)\right), \\
& \nabla\left(w_{k_{n}}\right)^{m} \rightarrow \nabla U^{m} \quad \text { weakly } \quad \text { in } L^{2}\left(\delta, T ; L^{2}\left(\mathbb{R}^{N}\right)\right)
\end{aligned}
$$

for any $p$ with $1<p<\infty$ and for any $T>0$. The above (5.22) and (5.23) are shown as follows:

From Lemma 5.3 , we see that $\left(w_{k}\right)^{m}$ is bounded in $H^{1}\left(\delta, T ; L^{2}\left(\mathbb{R}^{N}\right)\right) \cap L^{\infty}\left(\delta, T ; H^{1}\left(\mathbb{R}^{N}\right)\right)$ for any $T>0$ and $\delta \in(0, T)$. Therefore, we can extract a subsequence such that

$$
\left(w_{k_{n}}\right)^{m} \rightarrow \xi \quad \text { strongly } \quad \text { in } C\left((\delta, T) ; L_{l o c}^{2}\left(\mathbb{R}^{N}\right)\right)
$$


for any $T>0$ and $\delta \in(0, T)$. This gives

$$
\left(w_{k_{n}}\right)^{m}(x, t) \rightarrow \xi(x, t) \quad \text { a.a } \quad x \in \mathbb{R}^{N}, t \in(0, T) .
$$

A function $g(w)=w^{\frac{1}{m}}$ is continuous with respect to $u$.

Thus, we see that

$$
w_{k_{n}}(x, t) \rightarrow \xi^{\frac{1}{m}}(x, t) \quad \text { a.a } \quad x \in \mathbb{R}^{N}, t \in(0, T) .
$$

On the other hand, by Lemma 5.2 , we see that there exists a constant $W_{\delta, T}$ independently of $k$ such that

$$
\sup _{\delta<t<T}\left\|w_{k}(t)\right\|_{L^{\infty}} \leq W_{\delta, T}
$$

Therefore, Lebesgue dominated convergence theorem and (5.21) gives that

$$
w_{k_{n}} \rightarrow \quad \xi^{\frac{1}{m}}=U \quad \text { strongly } \quad \text { in } L^{p}\left(\delta, T ; L_{l o c}^{p}\left(\mathbb{R}^{N}\right)\right)
$$

for any $p \in(1, \infty)$. From $(5.26)$, we observe that

$$
w_{k_{n}}(x, t) \rightarrow \xi^{\frac{1}{m}}(x, t)=U(x, t) \quad \text { a.a. } x \in \mathbb{R}^{N} \text {, all } t \in(0, T) .
$$

From (5.24) and (5.27),

$$
w_{k_{n}}^{m} \rightarrow U^{m} \quad \text { strongly } \quad \text { in } C\left((\delta, T) ; L_{l o c}^{2}\left(\mathbb{R}^{N}\right)\right) \text {. }
$$

In addition, since $|b-a|^{m} \leq\left|b^{m}-a^{m}\right|$ for $0 \leq a \leq b$ and $m \geq 1$, from (5.22) we see that

$$
w_{k_{n}} \rightarrow U \quad \text { strongly } \quad \text { in } C\left((\delta, T) ; L_{l o c}^{2 m}\left(\mathbb{R}^{N}\right)\right)
$$

By Hölder inequality and (5.29), in all cases of $1<p<\infty$, it holds that

$$
w_{k_{n}} \rightarrow U \quad \text { strongly } \quad \text { in } C\left((\delta, T) ; L_{l o c}^{p}\left(\mathbb{R}^{N}\right)\right)
$$

for any $T>0$ and $\delta \in(0, T)$. Now let $\delta \rightarrow 0$. Employing a diagonal process,

$$
w_{k_{n}} \rightarrow U \quad \text { strongly } \quad \text { in } C\left((0, T) ; L_{l o c}^{p}\left(\mathbb{R}^{N}\right)\right)
$$

which prove (5.22). From (5.14) in Lemma 5.3 and (5.30), we obtain (5.23).

Using (5.22)-(5.23), we find by $q>m+\frac{2}{N}$ that $U(x, t)$ satisfies the porous medium equation in a distribution sense, i.e., that

$$
\int_{\tau_{1}}^{\tau_{2}} \int U \varphi_{t}+U^{m} \Delta \varphi d x d t=\int U\left(x, \tau_{2}\right) \varphi\left(x, \tau_{2}\right) d x-\int U\left(x, \tau_{1}\right) \varphi\left(x, \tau_{1}\right) d x
$$

for all $C^{2}$ functions $\varphi(x, t)$ with compact support in $\mathbb{R}^{N} \times(0, T]$, and all $0<\tau_{1}<\tau_{2}<T$. We now remark that the critical case of $q=m+\frac{2}{N}$ should be excluded from Theorem 1.2 . 


\subsection{Key lemma}

We are now in a position to prove the following key lemma:

Lemma 5.4 (key lemma). Let $N \geq 1, m>1$ and $q \geq \max \left\{m+\frac{2}{N}, 2\right\}$. Let $\left(w_{k}, z_{k}\right)$ be a weak solution of ${ }^{w}(\mathrm{KS})$ and let $U$ satisfy (5.31). We assume that $w_{k}$ converges to $U$ in $C\left((0, T) ; L_{\text {loc }}^{p}\left(\mathbb{R}^{N}\right)\right)$ with $1 \leq p<\infty$. Then, it holds that

$$
\left|\int_{\mathbf{R}^{N}}\left(w_{k}(x, t)-U(x, t)\right) \mathrm{d} x\right| \rightarrow 0 \quad \text { a.a. } t \in(0, T) \quad \text { as } k \rightarrow \infty
$$

Proof of Lemma 5.4. It is easy to verify that $w_{k} \rightarrow U$ strongly in $L_{l o c}^{1}\left(\mathbb{R}^{N}\right)$. Indeed, it holds that

$$
\left\|w_{k_{n}}(t)-U(t)\right\|_{L^{1}(K)} \leq\left\|w_{k_{n}}(t)-U(t)\right\|_{L^{p}(K)}^{\frac{1}{p}}|K|^{\frac{p-1}{p}} \rightarrow 0 \quad \text { all } t \in(0, T)
$$

as $k_{n} \rightarrow \infty$ for any compact set $K \subset \subset \mathbb{R}^{N}$ and for any $T>0$.

Next, we fix a time $t$ in $(0, \infty)$ and prove that for any $\lambda>0$, there exist $f_{\lambda}(\cdot, t) \in L^{1}\left(\mathbb{R}^{N}\right)$ and $k_{0} \in \mathbb{N}$ such that

$$
\int\left[w_{k}(x, t)-f_{\lambda}(x, t)\right]^{+} \mathrm{d} x<\frac{\lambda}{6} \quad \text { for any } k>k_{0} .
$$

To this end, we prepare the following lemma:

Lemma 5.5. Let $N \geq 1, m>1$ and $q \geq \max \left\{m+\frac{2}{N}, 2\right\}$. Let $(u, v)$ be the weak solution of (KS) obtained in Theorem 1.1 and let $U$ satisfy (5.31). Then, there exists a function $g \in L^{1}(0, \infty)$ such that

$$
\begin{array}{r}
\int_{\mathbf{R}^{N}}\left[u\left(t_{2}\right)-U\left(t_{2}\right)\right]^{+} \mathrm{d} x \mathrm{~d} t-\int_{\mathbf{R}^{N}}\left[u\left(t_{1}\right)-U\left(t_{1}\right)\right]^{+} \mathrm{d} x \mathrm{~d} t \leq \int_{t_{1}}^{t_{2}} g(t) \mathrm{d} t \\
\text { for all } t_{1}, t_{2} \text { with } 0<t_{1}<t_{2}<\infty .
\end{array}
$$

Proof of Lemma 5.5. We give the formal calculation. $\eta_{n}(r)$ is a sequence of cut-off functions defined by $\eta_{n}(r):=$ $\eta(n t)$ with $\eta$ defined in Lemma 2.3. By multiplying the first equation in (KS) by $\eta_{n}\left(u^{m}-U^{m}\right)$ and integrating it over $\mathbb{R}^{N}$, we get

$$
\begin{aligned}
\int_{\mathbf{R}^{N}}(u-U)_{t} \cdot \eta_{n}\left(u^{m}-U^{m}\right) \mathrm{d} x= & -\int_{\mathbb{R}^{N}}\left|\nabla\left(u^{m}-U^{m}\right)\right|^{2} \cdot \eta_{n}^{\prime}\left(u^{m}-U^{m}\right) \mathrm{d} x \\
& -\int_{\mathbb{R}^{N}}\left(\nabla u^{q-1} \cdot \nabla v+u^{q-1} \cdot \Delta v\right) \cdot \eta_{n}\left(u^{m}-U^{m}\right) \mathrm{d} x \\
\leq & -\int_{\mathbb{R}^{N}}\left(\nabla u^{q-1} \cdot \nabla v \cdot \eta_{n}\left(u^{m}-U^{m}\right)+u^{q} \cdot \eta_{n}\left(u^{m}-U^{m}\right)\right) \mathrm{d} x \\
\leq & \int_{\mathbf{R}^{N}}\left((q-1) u^{q-2}|\nabla u||\nabla v|+u^{q}\right) \mathrm{d} x \\
\leq & \int_{\mathbf{R}^{N}} u^{q-2}|\nabla u|^{2} \mathrm{~d} x+c_{q} \int_{\mathbf{R}^{N}}\left(u^{q}+|\nabla v|^{q}\right) \mathrm{d} x \\
\leq & \int_{\mathbf{R}^{N}} u^{q-2}|\nabla u|^{2} \mathrm{~d} x+\hat{c_{q}} \int_{\mathbf{R}^{N}} u^{q} \mathrm{~d} x=: I+J
\end{aligned}
$$


where

$$
I(t):=\int_{\mathbb{R}^{N}} u^{q-2}|\nabla u|^{2}(t) \mathrm{d} x, \quad J(t):=\hat{c_{q}} \int_{\mathbf{R}^{N}} u^{q}(t) \mathrm{d} x
$$

and $c_{q}$ and $\hat{c_{q}}$ are constants depending only on $q$.

We are now going to show that $I, J \in L^{1}(0, \infty)$. Similarly to $(4.1)$, we have

$\|u(t)\|_{L^{r}}^{r}+m r(r-1) \int_{0}^{t} \int_{\mathbb{R}^{N}} u^{r+m-3}|\nabla u|^{2} \mathrm{~d} x \mathrm{~d} s \leq\left\|u_{0}\right\|_{L^{r}}^{r}+\frac{r(r-1)}{r+q-2} \int_{0}^{t}\|u(s)\|_{L^{r+q-1}}^{r+q-1} \mathrm{~d} s \quad$ for all $r \in(1, \infty)$.

Taking $r$ by $r=q-m+1\left(>1+\frac{2}{N}\right)$ in (5.37), from Theorem 1.1 and $q \geq m+\frac{2}{N}>\left(m+\frac{1}{N}\right)$, we have

$$
\begin{aligned}
\int_{0}^{\infty} I(t) \mathrm{d} t & \leq c_{m, q}\left\|u_{0}\right\|_{L^{q-m+1}}^{q-m+1}+c_{m, q} \int_{0}^{\infty}\|u(s)\|_{L^{2 q-m}}^{2 q-m} \mathrm{~d} s \\
& \leq c_{m, q}\left\|u_{0}\right\|_{L^{q-m+1}}^{q-m+1}+C_{2} \int_{0}^{\infty}(1+t)^{\frac{-\frac{N}{N(m-1)+2} \cdot(2 q-m-1)}{\mathrm{d}} \mathrm{d} t} \\
& \leq c_{m, q}\left\|u_{0}\right\|_{L^{q-m+1}}^{q-m+1}+C_{3}
\end{aligned}
$$

where $c_{m, q}$ is a constant depending only on $m, q$ and $C_{2}$ and $C_{3}$ are constants depending only on $m, q, N,\left\|u_{0}\right\|_{L^{1}}$ and $\left\|u_{0}\right\|_{L^{2 q-m}}$.

By Theorem 1.1 and $q \geq m+\frac{2}{N}$, similarly to (5.38), we have

$$
\int_{0}^{\infty} J(t) \mathrm{d} t \leq \hat{c_{q}} \int_{0}^{\infty}(1+t)^{-\frac{N}{N(m-1)+2} \cdot(q-1)} \mathrm{d} t<C_{4}
$$

where $C_{4}$ depends only on $m, q, N,\left\|u_{0}\right\|_{L^{1}}$ and $\left\|u_{0}\right\|_{L^{q}}$.

We set $[s]^{+}=\max (s, 0)$ and

$$
\begin{cases}\operatorname{sign}_{0}^{+}(s)=1 & \text { for } s>0 \\ \operatorname{sign}_{0}^{+}(s)=0 & \text { for } s \leq 0\end{cases}
$$

Noting that $\eta_{n}$ converge to the sign function $\operatorname{sign}_{0}^{+}$as $n \rightarrow \infty$ and $\partial_{t}[u-U]^{+}=\partial_{t}(u-U) \cdot \operatorname{sign}_{0}^{+}(u-U)$. $(\operatorname{See}$ Gilbarg-Trudinger [14].)

Taking the limit in (5.36) as $n \rightarrow \infty$ and integrating it from $t_{1}$ to $t_{2}$, we have

$$
\int\left[u\left(t_{2}\right)-U\left(t_{2}\right)\right]^{+} \mathrm{d} x-\int\left[u\left(t_{1}\right)-U\left(t_{1}\right)\right]^{+} \mathrm{d} x \leq \int_{t_{1}}^{t_{2}} I(s)+J(s) \mathrm{d} s<\infty
$$

for any $t_{1}, t_{2}$ with $0<t_{1} \leq t_{2}<\infty$. Thus, we observe that there exists $g=(I+J) \in L^{1}(0, \infty)$ satisfying (5.35). Thus, we complete the proof of Lemma 5.5.

Let us define $\beta$ and $V(x, t ; M)$ by

$$
\begin{aligned}
\int_{\mathbb{R}^{N}}\left(\beta^{2}-\frac{m-1}{2 m(N(m-1)+2)} \cdot|y|^{2}\right)_{+}^{\frac{1}{m-1}} \mathrm{~d} y & =1 \text { and } \\
V(x, t ; M) & :=\frac{1}{t^{\frac{N}{N(m-1)+2}}}\left(\beta^{2} M^{\frac{2(m-1)}{N(m-1)+2}}-\frac{m-1}{2 m(N(m-1)+2)} \cdot \frac{|x|^{2}}{t^{\frac{2}{N(m-1)+2}}}\right)_{+}^{\frac{1}{m-1}}
\end{aligned}
$$


Then, we easily see that $\int_{\mathbb{R}^{N}} V(x, t ; M) \mathrm{d} x=M$. It is known that the above function $V(x, t, M)$ is the exact solution of $u_{t}=\Delta u^{m}$ which is the so-called Barenblatt solution. (we refer to Barenblatt [2] for instance.) Therefore, we can take $U(x, t)$ by $U(x, t)=V(x, t ; M)$ in Lemma 5.5. Consequently, we see that for any $\lambda>0$, there exists $T_{\lambda}>0$ sufficiently large such that

$$
\sup _{T_{\lambda}<t<\infty}\left(\int_{\mathbb{R}^{N}}[u(x, t)-V(x, t ; M)]^{+} \mathrm{d} x-\int_{\mathbf{R}^{N}}\left[u\left(x, T_{\lambda}\right)-V\left(x, T_{\lambda} ; M\right)\right]^{+} \mathrm{d} x\right)<\frac{\lambda}{12} .
$$

Moreover, for any fixed $\lambda>0$, we can find a constant $M(\lambda)>0$ such that

$$
\int_{\mathbb{R}^{N}}\left[u\left(x, T_{\lambda}\right)-V\left(x, T_{\lambda} ; M(\lambda)\right)\right]^{+} \mathrm{d} x<\frac{\lambda}{12} .
$$

Indeed, for any $\lambda>0$, there exists a compact set $K_{\lambda} \subset \subset \mathbb{R}^{N}$ such that

$$
\begin{aligned}
\int_{\mathbf{R}^{N}}\left[u\left(x, T_{\lambda}\right)-V\left(x, T_{\lambda} ; M\right)\right]^{+} \mathrm{d} x & \leq \int_{\mathbf{R}^{N} \backslash K_{\lambda}}\left[u\left(x, T_{\lambda}\right)-V\left(x, T_{\lambda} ; M\right)\right]^{+} \mathrm{d} x+\int_{K_{\lambda}}\left[u\left(x, T_{\lambda}\right)-V\left(x, T_{\lambda} ; M\right)\right]^{+} \mathrm{d} x \\
& \leq \frac{\lambda}{12}+\int_{K_{\lambda}}\left[u\left(x, T_{\lambda}\right)-V\left(x, T_{\lambda} ; M\right)\right]^{+} \mathrm{d} x .
\end{aligned}
$$

On the other hand,

$$
\operatorname{supp} V\left(\cdot, T_{\lambda} ; M\right)=\left\{x \in \mathbb{R}^{N} ;|x| \leq M^{\frac{m-1}{N(m-1)+2}} \cdot \beta\left(\frac{2 m(N(m-1)+2)}{m-1}\right)^{\frac{1}{2}} T_{\lambda}^{\frac{1}{N(m-1)+2}}\right\}
$$

and

$$
V\left(x, T_{\lambda} ; M\right)^{m-1}=M^{\frac{2(m-1)}{N(m-1)+2}} \cdot T_{\lambda}^{-\frac{N(m-1)}{N(m-1)+2}} \beta^{2}-\frac{m-1}{2 m(N(m-1)+2)} T_{\lambda}^{-1}|x|^{2} \quad \text { in supp } V\left(\cdot, T_{\lambda} ; M\right) .
$$

By Theorem 1.1, the weak solution $u$ belongs to $L^{\infty}\left(0, \infty ; L^{1} \cap L^{\infty}\left(\mathbb{R}^{N}\right)\right)$. Therefore, taking $M=M\left(\lambda, K_{\lambda}, T_{\lambda}\right)$ sufficiently large, we verify

$$
\int_{K_{\lambda}}\left[u\left(x, T_{\lambda}\right)-V\left(x, T_{\lambda} ; M\right)\right]^{+} \mathrm{d} x=0 .
$$

Combining (5.43) with (5.44), we obtain (5.42).

In consequence, from (5.41) and (5.42), we observe that there exist $T_{\lambda}>0$ and $M_{\lambda}>0$ such that

$$
\sup _{T_{\lambda}<t<\infty} \int_{\mathbf{R}^{N}}\left[u(x, t)-V\left(x, t ; M_{\lambda}\right)\right]^{+} \mathrm{d} x \leq \frac{\lambda}{6} .
$$

On the other hand, it is easy to see that $V\left(x, t ; M_{\lambda}\right)=k^{N} V\left(k x, k^{N(m-1)+2} t ; M_{\lambda}\right)$ all $x \in \mathbb{R}^{N}$ and $t \in(0, \infty)$. Therefore, the above (5.45) is equivalent to

$$
\sup _{\frac{T_{\lambda}}{t}<k^{N(m-1)+2}<\infty} \int_{\mathbf{R}^{N}}\left[w_{k}(x, t)-V\left(x, t ; M_{\lambda}\right)\right]^{+} \mathrm{d} x \leq \frac{\lambda}{6} .
$$


From (5.46), we see that for any fixed time $t \in(0, \infty)$ and $\lambda>0$, there exist $V\left(\cdot, t ; M_{\lambda}\right) \in L^{1}\left(\mathbb{R}^{N}\right)$ and $k_{0}=\left(\frac{T_{\lambda}}{t}\right)^{\frac{1}{N(m-1)+2}}$ such that

$$
\int_{\mathbb{R}^{N}}\left[w_{k}(x, t)-V\left(x, t ; M_{\lambda}\right)\right]^{+} \mathrm{d} x<\frac{\lambda}{6} \quad \text { for any } k>k_{0} .
$$

We thus conclude by Lemma 2.4 that

$$
\left|\int_{\mathbb{R}^{N}}\left(w_{k}(x, t)-U(x, t)\right) \mathrm{d} x\right| \rightarrow 0 \quad \text { as } k \rightarrow \infty
$$

for all $t \in(0, \infty)$.

\subsection{Proof of Theorem $\mathbf{1 . 2}$}

From (5.48), we see that

$$
\int U(1) \mathrm{d} x=\int u_{0}(x) \mathrm{d} x
$$

Indeed, by the mass conservation law $\left(\left\|w_{k}(t)\right\|_{L^{1}}=\left\|w_{k}(0)\right\|_{L^{1}}\right.$ for all $\left.t \geq 0\right)$ and (5.48), it holds that

$$
\int u_{0}(x) \mathrm{d} x=\int w_{k}(x, 0) \mathrm{d} x=\int w_{k}(x, t) \mathrm{d} x \rightarrow \int U(x, t) \mathrm{d} x \quad \text { for a.a. } t \in(0, \infty),
$$

which yields (5.48). On the other hand, by virtue of (5.22), (5.23), (5.31), $U$ satisfies that

$$
U(\cdot, 1) \in L_{l o c}^{1}\left(\mathbb{R}^{N}\right) \quad \text { and } \quad U^{m}, \nabla U^{m} \in L^{1}\left(1, T ; L_{l o c}^{1}\left(\mathbb{R}^{N}\right)\right)
$$

and

$$
\int_{1}^{\infty} \int\left(U \varphi_{t}+U^{m} \Delta \varphi\right) \mathrm{d} x \mathrm{~d} t=\int U(x, 1) \varphi(x, 1) \mathrm{d} x
$$

for all smooth functions $\varphi(x, t)$ with compact support in $\mathbb{R}^{N} \times[1, \infty)$. Therefore, by Theorem 1.1 in Vazquez [34] we obtain that

$$
t^{\frac{N}{N(m-1)+2}}\left\|U(\cdot, t)-V\left(\cdot, t ;\left\|u_{0}\right\|_{L^{1}\left(\mathbf{R}^{N}\right)}\right)\right\|_{L^{p}\left(\mathbf{R}^{N}\right)} \rightarrow 0 \quad \text { as } t \rightarrow \infty
$$

for any $p \in[1, \infty]$.

For any positive numbers $\varepsilon, R$, we define $B_{t}$ by

$$
B_{t}:=\left\{x \in \mathbb{R}^{N} ;|x|<R t^{\frac{1}{N(m-1)+2+\varepsilon}}\right\} .
$$

From (5.27), $U(x, t)$ is defined almost all $(x, t) \in \mathbb{R}^{N} \times(0, \infty)$. From $(5.22), w_{k}(x, t)$ converges to $U$ in $L_{l o c}^{p}\left(\mathbb{R}^{N}\right)(1<p<\infty)$ for all $t \in[1, \infty)$. Therefore, for any fixed positive number $\varepsilon$, taking $t=k^{\varepsilon} \geq 1$ and noting that all subsequence $\left\{w_{k_{n}}\right\}$ of $\left\{w_{k}\right\}$ have the subsequence $\left\{w_{k_{n^{\prime}}}\right\}$ of $\left\{w_{k_{n}}\right\}$ which converges to the Barenblatt solution, we see that

$$
\begin{aligned}
& \left\|w_{k}\left(\cdot, k^{\varepsilon}\right)-V\left(\cdot, k^{\varepsilon} ;\left\|u_{0}\right\|_{L^{1}}\right)\right\|_{L^{p}\left(B_{R}\right)} \\
& \leq\left\|w_{k}\left(\cdot, k^{\varepsilon}\right)-U\left(\cdot, k^{\varepsilon}\right)\right\|_{L^{p}\left(B_{R}\right)}+\left\|U\left(\cdot, k^{\varepsilon}\right)-V\left(\cdot, k^{\varepsilon} ;\left\|u_{0}\right\|_{L^{1}}\right)\right\|_{L^{p}\left(\mathbf{R}^{N}\right)} \\
& \rightarrow 0 \quad \text { as } k(\in \mathbb{R}) \rightarrow \infty
\end{aligned}
$$

for all $p \in(1, \infty)$. 
Since $V(x, t ; M)$ is a self-similar solution, from (5.54), we obtain

$$
k^{N\left(1-\frac{1}{p}\right)} \| u\left(\cdot, k^{N(m-1)+2+\varepsilon}\right)-V\left(\cdot, k^{N(m-1)+2+\varepsilon} ;\left\|u_{0}\right\|_{\left.L^{1}\right)} \|_{L^{p}\left(k B_{R}\right)} \rightarrow 0 \quad \text { as } k \rightarrow \infty\right.
$$

for any $\varepsilon>0$ and $p \in(1, \infty)$.

Taking $k$ by $k=t^{\frac{1}{N(m-1)+2+\varepsilon}}$ in $(5.55)$, we thus obtain

$$
t^{\frac{N}{N(m-1)+2+\varepsilon}\left(1-\frac{1}{p}\right)}\left\|u(\cdot, t)-V\left(\cdot, t ;\left\|u_{0}\right\|_{L^{1}}\right)\right\|_{L^{p}\left(B_{t}\right)} \rightarrow 0 \text { as } t \rightarrow \infty,
$$

for any $\varepsilon>0$ and $p \in(1, \infty)$ and for all $R>0$, where $B_{t}=B_{t}(\varepsilon, R)$ is the ball defined in (5.53).

Thus, we complete the proof of Theorem 1.2 for all cases of $p \in(1, \infty)$.

Acknowledgements. This article was written while the second author stayed at Max Planck Institute for Mathematics in Leipzig. The second author would like to express her sincere gratitude to Professor A. Stevens and all other members at MPI for their cordial hospitality. The second author also wishes to express her sincere gratitude to Professors T. Nagai and H. Kozono for many stimulating conversations and helpful advice.

\section{REFERENCES}

[1] N.D. Alikakos, $L^{p}$ bounds of solutions of reaction-diffusion equations. Comm. Partial Diff. Equ. 4 (1979) 827-868.

[2] G.I. Barenblatt, On some unsteady motions of a fluid and a gas in a porous medium. Prikl. Mat. Mekh. 16 (1952) 67-78.

[3] P.H. Bénilan, Opérateurs accrétifs et semi-groupes dans les espaces $L^{p}(1 \leq p \leq \infty)$. France-Japan Seminar, Tokyo (1976).

[4] P. Biler, T. Nadzieja and R. Stanczy, Nonisothermal systems of self-attracting Fermi-Dirac particles. Banach Center Pulb. 66 (2004) 61-78.

[5] P. Biler, M. Cannone, I.A. Guerra and G. Karch, Global regular and singular solutions for a model of gravitating particles. Math. Ann. 330 (2004) 693-708.

[6] H. Brezis, Analyse fonctionnelle, Theorie et applications. Masson (1983).

[7] S. Childress and J.K. Percus, Nonlinear aspects of chemotaxis. Math. Biosci. 56 (1981) 217-237.

[8] J.I. Diaz, T. Nagai and J.M. Rakotoson, Symmetrization techniques on unbounded domains: Application to a chemotaxis system on $\mathbb{R}^{N}$. J. Diffierential Equations 145 (1998) 156-183.

[9] J. Duoandikoetxea, Fourier Analysis, Graduate studies Mathematics 29 AMS, Providence, Rhode Island (2000).

[10] A. Friedman and S. Kamin, The asymptotic behavior of gas in an N-dimensional porous medium. Trans. Amer. Math. Soc. 262 (1980) 551-563.

[11] H. Fujita, On the blowing up of solutions of the Cauchy problem for $u_{t}=\Delta u+u^{1+\alpha}$. J. Fac. Sci. Univ. Tokyo Sect. I 13 (1966) 109-124.

[12] V.A. Galaktionov, Blow-up for quasilinear heat equations with critical Fujita's exponents. Proc. Roy. Soc. Edinburgh Sect. A 124 (1994) 517-525.

[13] V.A. Galaktionov and S.P. Kurdyumov, A.P. Mikhailov and A.A. Samarskiin, On unbounded solutions of the Cauchy problem for a parabolic equation $u_{t}=\nabla \cdot\left(u^{\sigma} \nabla u\right)+u^{\beta}$. Sov. Phys., Dokl. 25 (1980) 458-459.

[14] D. Gilbarg and N.S. Trudinger, Elliptic Partial Differential Equations of Second Order. Springer-Verlag, Berlin-New York (1983).

[15] M.A. Herrero and Juan J.L. Velázquez, Chemotactic collapse for the Keller-Segel model. J. Math. Biol. 35 (1996) 177-194.

[16] M.A. Herrero and Juan J.L. Velázquez, Singularity patterns in a chemotaxis model. Math. Ann. 306 (1996) 583-623.

[17] D. Horstmann, From 1970 until present: the Keller-Segel model in chemotaxis and its consequences. I. Jahresber. Deutsch. Math.-Verein. 105 (2003) 103-165.

[18] D. Horstmann, From 1970 until present: the Keller-Segel model in chemotaxis and its consequences. II. Jahresber. Deutsch. Math.-Verein. 106 (2004) 51-69.

[19] W. Jäger and S. Luckhaus, On explosions of solutions to a system of partial differential equations modelling chemotaxis. Trans. Amer. Math. Soc. 329 (1992) 819-824.

[20] S. Kamin, Similar solutions and the asymptotics of filtration equations. Arch. Rational Mech. Anal. 60 (1976) $171-183$.

[21] S. Kamin and J.L. Vazquez, Fundamental solutions and asymptotic behaviour for the p-Laplacian equation. Rev. Mat. Iberoamericana 4 (1988) 339-354.

[22] T. Kawanago, Existence and behavior of solutions for $u_{t}=\Delta\left(u^{m}\right)+u^{\ell}$. Adv. Math. Sci. Appl. 7 (1997) 367-400.

[23] E.F. Keller and L.A. Segel, Initiation of slime mold aggregation viewed as an instability. J. Theor. Biol. 26 (1970) $399-415$. 
[24] K. Mochizuki and R. Suzuki, Critical exponent and critical blow-up for quasilinear parabolic equations. Israel J. Math. 98 (1997) $141-156$.

[25] T. Nagai, T. Senba and K. Yoshida, Application of the Moser-Trudinger inequality to a parabolic system of chemotaxis. Funkc. Ekvacioj 40 (1997) 411-433.

[26] T. Nagai, R. Syukuinn and M. Umesako, Decay Properties and Asymptotic Profiles of Bounded Solutions to a Parabolic System of Chemotaxis in $\mathbb{R}^{N}$. Funkc. Ekvacioj 46 (2003) 383-407.

[27] M. Nakao, Global solutions for some nonlinear parabolic equations with nonmonotonic perturbations. Nonlinear Analysis, Theory, Method Appl. 10 (1986) 299-314.

[28] T. Senba and T. Suzuki, Local and norm behavior of blowup solutions to a parabolic system of chemotaxis. J. Korean Math. Soc. 37 (2000) 929-941.

[29] E.M. Stein, Singular integrals and differentiability properties of functions. Princeton Mathematical Series, No. 30 Princeton University Press, Princeton, N.J. (1970).

[30] Y. Sugiyama, Global existence and decay properties of solutions for some degenerate quasilinear parabolic systems modelling chemotaxis. Nonlinear Anal. 63 (2005) 1051-1062.

[31] Y. Sugiyama, Time global existence and asymptotic behavior of solutions to degenerate quasi-linear parabolic systems for chemotaxis-growth models, (submitted).

[32] Y. Sugiyama and H. Kunii, Global existence and decay properties for a degenerate Keller-Segel model with a power factor in drift term. J. Differential Equations (in press).

[33] Y. Sugiyama, Global existence in sub-critical cases and finite time blow-up in super-critical cases to degenerate Keller-Segel systems. Diff. Integral Equations, (to appear).

[34] J.L. Vazquez, Asymptotic behaviour for the porous medium equation posed in the whole space. J. Evol. Equ. 3 (2003) 67-118.

[35] L. Véron, Coercivité et propriétés régularisantes des semi-groupes nonlinéaires dans les espaces de Banach. Ann. Fac. Sci. Toulouse 1 (1979) 171-200.

To access this journal online:

www.edpsciences.org 